Part of Journal of Research of the National Bureau of Standards, Volume 23, December 1939

\title{
ZEEMAN EFFECT IN THE SECOND AND THIRD SPECTRA OF XENON
}

\author{
By Curtis J. Humphreys, William F. Meggers, and T. L. deBruin*
}

\section{ABSTRACT}

The Zeeman effect in the spectra of singly and doubly ionized xenon has been observed in the laboratory "Physica" in Amsterdam and at the National Bureau of Standards. The source used in the case of the former observations was a Back lamp consisting of a spark between metal electrodes operated in an atmosphere of the gas, whereas at the National Bureau of Standards a Geissler tube placed transversely between the poles of the magnet was employed. Measurable patterns were obtained for 130 lines of $\mathrm{Xe}$ II and 62 of Xe III. Reasonably good agreement between the observations from the two laboratories was obtained.

Analysis of observed patterns has permitted computation of $61 \mathrm{~g}$ values associated with levels of $\mathrm{Xe}$ II and 34 of Xe rir.

Most of the patterns are only partially resolved. Use of the formulas of Shenstone and Blair to set up observation equations which were adjusted by the method of least squares has permitted calculation of a fairly consistent set of $g$ values. The $g$-sum rule was tested where possible and was found to be satisfied very closely in case of two different electron configurations in $\mathrm{Xe}^{+}$. Observed $g$ values depart considerably from those calculated by the Landé formula in most instances. A vector coupling scheme intermediate between $L S$ and $J j$ is indicated for both $\mathrm{Xe}^{+}$and $\mathrm{Xe}^{++}$.

The analysis of $\mathrm{Xe}$ II has not required important changes as a result of the Zeeman-effect observations. No changes of $J$ values are indicated. In two instances, pairs of levels are interchanged in the term tables. In Xe III, the revisions are confined mostly to the group of levels of ${ }^{2} \mathrm{D}$ parentage from the $s^{2} p^{3} \cdot 6 p$ configuration. Changes in the assignment of $J$ values are required in five instances.

\section{CONTENTS}

I. Introduction

II. Observations_........

III. Calculation and interpretation of the $g$ factors $\ldots \ldots .685$

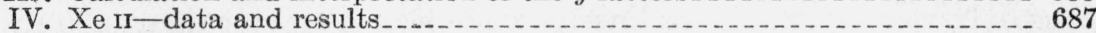

V. Xe III-data and results

VI. References.

\section{INTRODUCTION}

A series of papers dealing with the analyses of the spectra originating in singly and in doubly ionized xenon have been published jointly or separately by the authors of this paper. A partial analysis of Xe II was published in 1931 [1]. ${ }^{1}$ In a recent paper [2] this analysis is revised and extended so that nearly all conspicuous lines of $\mathrm{Xe}$ II are accounted for. DeBruin announced the first-observed regularities in Xe III in 1935 [3]. Humphreys [4], in 1936, extended the analysis to

* Professor of Physies, Laboratory "Physica," University of Amsterdam, Amsterdam, Holland.

1 Numbers in brackets indicate the literature references at the end of this paper. 
account for most of the observed lines. Portions of these spectra in the Schumann region have been observed and analyzed by Boyce [5].

The second, third, and probably higher spectra of xenon are excited simultaneously in the sources commonly used, such as the electrodeless discharge or a Geissler tube operated in a circuit containing a spark gap and condensers. Methods of identifying a particular spectrum or of favoring its production are discussed in the literature referred to. Lines of the third, or higher, spectra appearing in the Geissler-tube source are weakened or suppressed, by inserting suitable amounts of inductance in the discharge circuit.

The earlier work of the authors, as well as that of Bloch, Bloch, and Déjardin $[6,7]$ has made it possible to classify almost all xenon lines according to the ions in which they originate.

A few Zeeman patterns in Xe III were available to deBruin when he published his paper on the Spectral Structure of the Doubly Ionized Noble Gases [3]. These had been observed by C. J. Bakker at the laboratory "Physica" in Amsterdam, but the results had not been published. The other investigations of the structure of $\mathrm{Xe}$ II and Xe III were carried out without the aid of the Zeeman effect.

\section{OBSERVATIONS}

Early in 1938 deBruin communicated to the other authors of this paper a set of Zeeman effect observations in xenon spectra in the region between 3400 and $5600 \mathrm{~A}$. These observations included about 60 patterns for $\mathrm{Xe}$ II and 20 for $\mathrm{X}$ e III. A number of additional patterns were indicated as "provisional." These have been incorporated into the tables in cases where interpretation of the patterns gave results consistent with other observations.

A year later, Zeeman effect observations in $\mathrm{Xe}$ Ir and $\mathrm{Xe}$ IIr were made at the National Bureau of Standards. These observations duplicated in part the earlier work but extended farther into the red to about $7000 \mathrm{~A}$, and yielded measurable patterns of several intense low-level lines of $\mathrm{Xe}$ II not observed by deBruin. Ninety-seven patterns of $\mathrm{Xe}$ II and 62 of Xe III were measured. Most of the patterns were recorded more than once, a few as many as four times. A 30,000-line/inch grating was used for all the observations at the National Bureau of Standards. The ultraviolet lines were observed in both the first and second order spectra, whereas first order observations only were available for lines of wave length greater than $4250 \mathrm{~A}$. The essential difference between the Amsterdam and Washington observations was in the sources employed. DeBruin used a Back lamp [8], which consists of a chamber containing the noble gas at low pressure mounted between the magnetic poles. A metallic spark is passed through the narrow space between the pole pieces. The spectra of the gas are excited along with the spectrum from the electrodes. This type of source probably yields sharper patterns but has the disadvantage of low intensity, making it necessary to maintain the magnet in operation for very long periods of time.

Geissler-tube sources were used in all of the observations at the National Bureau of Standards, the intensities of these sources permitted satisfactory spectrograms to be obtained in exposure times ranging from 30 to 60 minutes but the tubes, mounted with the capillary portion between the pole pieces and at right angles to the field, quickly became unserviceable because the discharge, driven 
against the capillary wall by the magnetic field darkened or caused disintegration of the tube wall. Tubes designed to be inserted in bored pole pieces to permit passage of the discharge parallel to the field were not used, because, according to Back [8], it is not possible to get a uniform field in the region of observation between bored pole pieces. A current of 150 amperes was maintained in the magnet windings by means of a motor generator. The applied electromotive force was about 100 volts. The field strength calculated from standard patterns of sodium, zinc, magnesium, and aluminum amounted to very nearly 36,000 gausses.

The greatest disadvantage of the use of condensed discharges from Geissler tubes is that the lines are not very sharp. Only in case of transitions between levels of small $J$ values, $1 / 2$ to $1 / 2$ or $1 / 2$ to $1 \frac{1}{2}$ in a spectrum of even multiplicity, such as Xe II, are completely resolved patterns obtainable. Resolution of the parallel components was obtained in only a few instances.

\section{CALCULATION AND INTERPRETATION OF THE g FACTORS}

The theoretical interpretation of the so-called anomalous Zeeman effect is too well known to require any extended discussion. The observed pattern results from the combination of levels, one or both of which are separated into a number of components by a magnetic field. These level displacements are accounted for by the acquisition of quantized amounts of energy $M g \mathrm{~L}$ in which $M$, the magnetic quantum number, stands for the quantized values of the projection of the vector $J$ upon the vector $H$. There are $2 J+1$ values of $M$, increasing by steps of one from $-J$ to $+J$. At the very beginning of the development of the quantum theory, Landé [9] set up a formula for the $g$ factor. It was later shown that this $g$ was the ratio of the magnetic to the mechanical angular momentum of the atom. $\mathbf{L}$ is the Lorentz unit ${ }^{2}$ given by $H e / 4 \pi m c^{2}$. It expresses the splitting which occurs in the normal Zeeman triplet. It is customary to specify all patterns in terms of these units of normal Zeeman splitting. The displacements of the components of a level are given then by the various products, $M g$, where $M$ takes all permitted values.

In cases where the coupling between the quantum vectors is of the LS type, a completely resolved Zeeman pattern may be analyzed to yield the quantum numbers, $J, L$, and $S$, associated with each of the combining levels.

It becomes apparent, therefore, that Zeeman-effect observations are extremely helpful in the assignment of quantum numbers to the levels of a spectrum. Complete tables of theoretical Zeeman patterns of the Landé type have been prepared by Kiess and Meggers [10] for transitions between levels belonging to terms of all multiplicities which may be expected to occur. Identification of any pattern by aid of the tables establishes the term transition responsible for the line as well as the $g$ factor for each level.

If the coupling is not of the $L S$ type, the splitting factors, or $g$ values, may still be derived from observed patterns if the $J$ 's of the combining levels are known. If more than one pattern involving the same level is observed, it is generally possible to determine an un-

\footnotetext{
The symbol $m$ in this expression refers to the mass of the electron. It is not used in this sense elsewhere in this paper.
} 
known $J$. Characteristic distributions of intensities of components in the pattern are shown, depending on whether the combining $J$ 's are equal or unequal. These features are easily recognized by the experienced observer. The solution of simultaneous linear equations involving the $g$ 's and magnetic quantum numbers of combining levels was first illustrated by Back [11] and is also discussed in the paper by Kiess and Meggers just referred to [10].

Theoretical $g$ values may be calculated by the following formula, in cases of $J j$-coupling:

$$
g=g_{i} \frac{J(J+1)+j_{i}\left(j_{i}+1\right)-J_{p}\left(J_{p}+1\right)}{2 J(J+1)}+g_{p} \frac{J(J+1)+J_{p}\left(J_{p}+1\right)-j_{i}\left(j_{i}+1\right)}{2 J(J+1)}
$$

The subscripts $i$ and $p$ refer, respectively, to the added electron and the parent configuration. It is not always possible to select the values of $j_{i}$ and $J_{p}$ which combine to give $J$ for a particular level. One can, however, tabulate the complete set, calculate the $g$ 's and then make the most reasonable correlation with the observed g's. One is required to make some assumption regarding $g_{p}$. If the parent level has $L S$ coupling, it is found at once from the Landé formula. This assumption has been made in our calculations, because the parent levels are the lowest in doubly ionized xenon. Zeeman effects cannot be observed for lines involving these levels, because they appear in the extreme-ultraviolet region, but in spectra where $g$ values have been determined for the lowest states, they are always of the Landé type. Other possibilities are that the parent level may have pure $J j$ coupling, or some type of intermediate coupling. The $g$ values for $s^{2} p^{4}$, on the assumption of pure $J j$ coupling are the same as for $s^{2} p^{2}$ and may be obtained by the above formula using the $J$ 's for two $p$ electrons. Calculation of $g$ 's, on the assumption of pure $J j$ coupling for the parent levels, were made for some of the levels of Xe II but are not included in the tables, because the results indicated this assumption to be less probable than that of $L S$ coupling for the normal configuration of the $\mathrm{X}^{++}$ion. It is probable that the coupling is actually of intermediate type as is to be expected in spectra of heavier noble gases. The matter will be discussed further when the results are treated in detail. Other observers have had considerable success in the calculation of $g$ 's for cases of intermediate coupling, and in the interpretation of observed patterns. An excellent example is the work of Green on the first spectra of noble gases [12].

Determination of a complete set of $g$ values, for the levels arising from a given electron configuration, permits the application of the $g$-sum rule [13]. According to this rule, the sum of the $g$ 's, associated with levels from a particular configuration, which have the same $J$ value, is independent of the coupling scheme. This sum may be obtained, of course, by adding the Landé $g$ 's for the levels of given $J$ predicted by the Hund theory for that configuration. Application of the rule is extremely useful in determining parentages and electron configurations associated with the levels of a spectrum.

In the present investigation, the problem of determining $g$ values was of considerable difficulty because not only was the vector coupling not of the $L S$ type, but most of the patterns were only partially 
resolved. Fortunately, the previous analysis of XeII and Xeri had given a generally correct assignment of $J$ values. For the calculation of $g$ values of partially resolved patterns, one must resort to the formulas of Shenstone and Blair [14]. These formulas are based on the theoretical distribution of intensities of components calculated by Hönl [15], and express the displacement of the center of intensity of an unresolved group of components in terms of the $g$ 's and $J$ 's of the combining levels. The formulas are of comparatively simple form. It turns out that, where $\Delta M=1$, the center of intensity of an unresolved group of $n$ components is one-fourth the distance from the strongest to the weakest component. Where $\Delta M=0$, the pattern of $n$ components is symmetrical about its own center, so that twice the measured displacement of an unresolved $n$ component gives directly the sum of the two $g$ 's. Shenstone and Blair remark that the use of their formulas is most satisfactory for term transitions involving nearly equal $g$ 's, especially in cases where the pattern is rather compact, and far from complete resolution. It is apparent, of course, that such formulas can be correct only to the extent that the actual intensity distribution in the pattern is the same as that theoretically predicted. We assume this to be true in employing the formulas, although the rather large departure from $L S$ coupling in these spectra may result in intensity distributions among the Zeeman components somewhat different from those given by the Hönl formulas. In the analysis of nearly resolved open patterns, it is sometimes best to assume that the measured positions of the components are the actual positions of the strongest components.

The Shenstone and Blair formulas may be applied if one of the $g$ 's is known and if we have the distance of the center of intensity of either the $n$ or $p$ components from the undisplaced line position, or if both $p$ and $n$ components are partially resolved, giving two equations involving the respective $g$ 's. In the latter case, it is necessary to decide which $g$ to assign to its respective level, if both are previously unknown. Usually a given level appears in a considerable number of transitions. This makes possible the writing of systems of simultaneous equations involving several observed patterns. Using the methods of least-squares adjustments, these observation equations may be transformed into normal equations and be solved to give the most probable set of $g$ 's. It is quite possible to adjust the entire set of observations in one normal solution. Because of the length and complexity of such an operation, it was found simpler in this investigation to make several small adjustments, each involving not more than about six observation equations.

\section{Xe II-DATA AND RESULTS}

All observed patterns in XeIr which were satisfactory for measurement are listed in table 1 . Here are shown in successive columns the wave lengths, wave numbers, term combinations, Zeeman patterns observed by Humphreys and Meggers, Zeeman patterns observed by deBruin, and the Zeeman patterns, calculated from the set of $g$ values, which appeared to be most consistent from the data as a whole. 
TABLE 1.-Zeeman patterns in Xe II

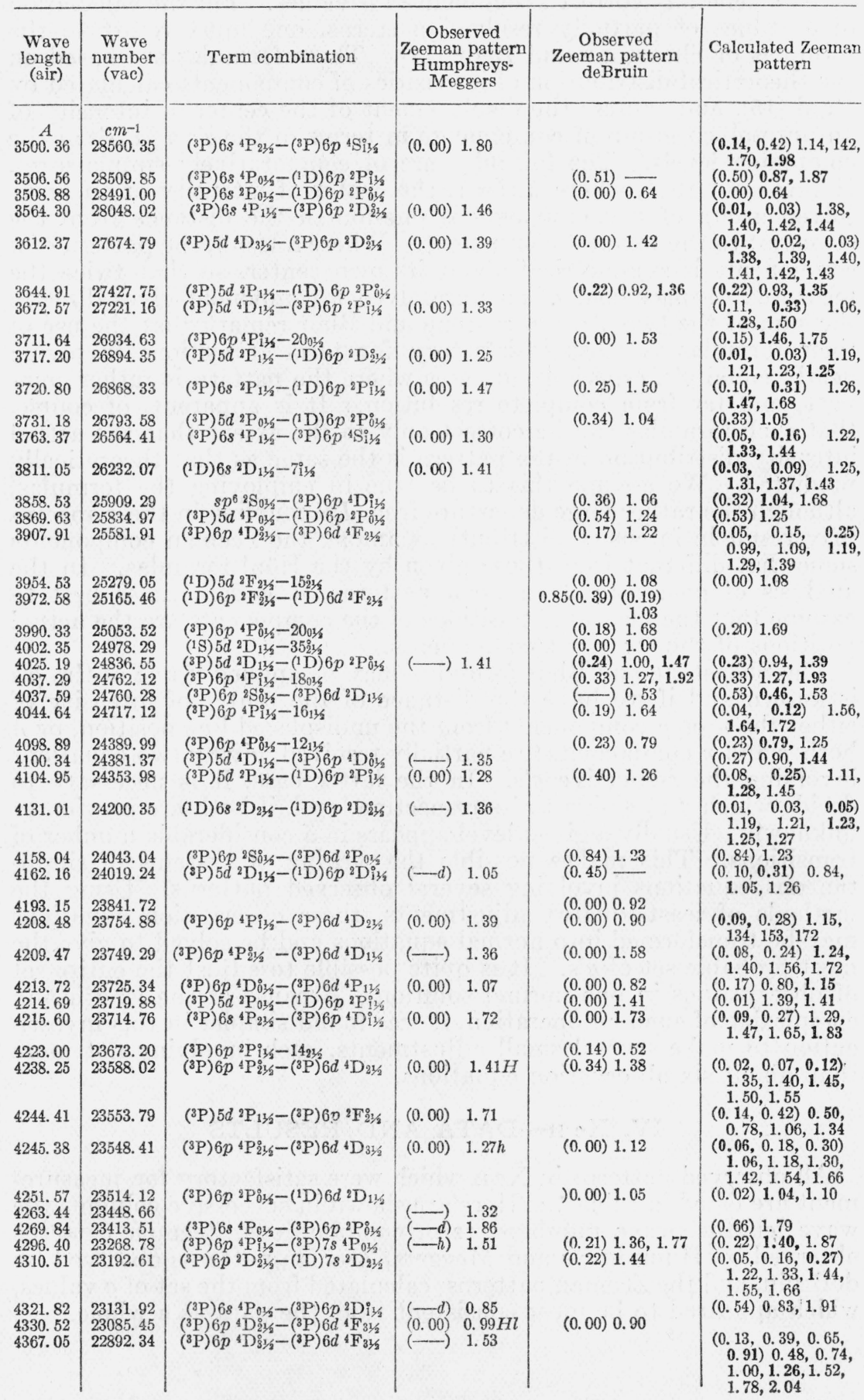


TABLE 1.-Zeeman patterns in Xe II-Continued

\begin{tabular}{|c|c|c|c|c|c|c|}
\hline $\begin{array}{l}\text { Wave } \\
\text { length } \\
\text { (air) }\end{array}$ & $\begin{array}{l}\text { Wave } \\
\text { number } \\
\text { (vac) }\end{array}$ & Term combination & $\begin{array}{r}\text { Obse } \\
\text { Zeeman } \\
\text { Hump } \\
\text { Mes }\end{array}$ & $\begin{array}{l}\text { n pattern } \\
\text { phreys- } \\
\text { ggers }\end{array}$ & $\begin{array}{c}\text { Observed } \\
\text { Zeeman pattern } \\
\text { deBruin }\end{array}$ & $\begin{array}{c}\text { Calculated Zeeman } \\
\text { pattern }\end{array}$ \\
\hline $\begin{array}{c}A \\
4369.20\end{array}$ & $\begin{array}{c}\mathrm{cm}^{-1} \\
22881.08\end{array}$ & P) $6 p^{4}$ & $(\longrightarrow)$ & & 2) 1.23 & 22 \\
\hline 4384.93 & 22799.00 & $s p^{6}{ }^{2} \mathrm{~S}_{01 / 2}-\left({ }^{3} \mathrm{P}\right) 6 p{ }^{4} \mathrm{P}^{\circ} 0_{1 / 2}$ & $(0.00)$ & 1.77 & $(0.28) 1.71$ & $(0.24) 1.76$ \\
\hline 4393. 20 & 22756.08 & ( $\left.{ }^{3} \mathrm{P}\right) 6 p^{4} \mathrm{~S}_{11 / 2}-\left({ }^{3} \mathrm{P}\right) 6 d^{2} \mathrm{D}_{21 / 2}$ & & & $(0.00) 1.13$ & $\begin{array}{c}(0.02,0.06) \\
1.17,1.21,1.25\end{array}$ \\
\hline 4395. 77 & 22742.77 & (1D) $5 d^{2} \mathrm{~F}_{21 / 2}-43_{31 / 2}^{\circ}$ & $=$ & & $(0.00) 1.04$ & $\begin{array}{l}(0.01,0.03,0.05) \\
1.01,1.03,1.05,\end{array}$ \\
\hline 4414. 84 & 22644.54 & ( $\left.{ }^{3} \mathrm{P}\right) 5 d^{2} \mathrm{D}_{21 / 2}-\left({ }^{1} \mathrm{D}\right) 6 p^{2} \mathrm{D}_{21 / 3}^{0}$ & $(0.29)$ & 1. 28 & $(0.27) 1.24$ & $\begin{array}{l}(0.06,0.18,0.30) \\
\quad 1.04,1.16,1.28 \\
1.40,1.52\end{array}$ \\
\hline 4448. 13 & 22475.07 & & $(0.00)$ & $1.14 H I$ & $(0.00) 1.09$ & \\
\hline $\begin{array}{l}4462.19 \\
4470.90\end{array}$ & $\begin{array}{l}22404.25 \\
22360.61\end{array}$ & ( $\left.{ }^{3 \mathrm{P}}\right) 5 d^{2} \mathrm{D}_{21 / 3}-\left({ }^{1} \mathrm{D}\right) 6 p^{2} \mathrm{D}_{1 / 5}{ }^{2}$ & $(0.00)$ & $\begin{array}{l}1.79 h \\
\text { 1. } 79 h\end{array}$ & (0. & $(0.19,0.58) \quad 0.75$ \\
\hline 4480.86 & 22310.90 & ( $\left.{ }^{3} \mathrm{P}\right) 6 p{ }^{4} \mathrm{D}_{1 / 2}{ }^{1}-\left({ }^{3} \mathrm{P}\right) 6 d^{4} \mathrm{~F}_{21 / 2}$ & & & $(0.00) 0.88$ & $(0.11,0.34), 0.78$ \\
\hline 4524. 21 & 22097.13 & ('P) $6 s^{4} \mathrm{P}_{01 / 2}-\left({ }^{3} \mathrm{P}\right) 6 p^{2} \mathrm{P}_{11 / 2}$ & $(0.58) 0$ & $0.79,1.92$ & $(0.54) 0.74,1.83$ & $(0.53) 0.86,1.92$ \\
\hline 4532.49 & 22056.76 & (iD) 6 & $(0.00)$ & & $(0$ & 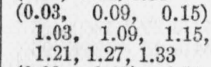 \\
\hline 4540.89 & 22015.96 & $\left.{ }^{3} \mathrm{P}\right) 6 p{ }^{2} \mathrm{D}_{1 / 2} \mathrm{~s}-\left({ }^{1} \mathrm{D}\right) 7 \mathrm{~s}^{2} \mathrm{D}_{21 / 2}$ & $(0.00)$ & 1. 22 & $(0.00) 1.23$ & $\begin{array}{c}(0.03,0.10) \quad 1.19, \\
1.26,1.33,1.40\end{array}$ \\
\hline 4545. 23 & 21994.94 & $\left({ }^{3} \mathrm{P}\right) 6 p^{4} \mathrm{D}_{23 / 2}^{0}-\left({ }^{3} \mathrm{P}\right) 6 d^{4} \mathrm{D}_{31 / 2}$ & $(0.00)$ & 1.62 & $(0.00) 1.47$ & $\begin{array}{l}(0.05, \quad 0.16, \quad 0.27) \\
1.08,1.19, \quad 1.30, \\
1.41,1.52,1.63\end{array}$ \\
\hline $\begin{array}{l}4555.94 \\
4577.06\end{array}$ & $\begin{array}{l}21943.24 \\
21841.98\end{array}$ & $\begin{array}{l}\left({ }^{3} \mathrm{P}\right) 6 p^{4} \mathrm{D}_{1 / 1}^{0}-20_{01 / 2} \\
\left.\left({ }^{3} \mathrm{P}\right) 6 p^{4} \mathrm{D}_{31 / 2}^{3}-{ }^{3} \mathrm{P}\right) 6 d^{4} \mathrm{D}_{21 / 2}\end{array}$ & $(0.00)$ & 1. 29 & $(0.23) 1.13$ & $\begin{array}{l}(0.26) 1.09,1.62 \\
(0.02, \quad 0.06, \quad 0.10) \\
1.29,1.33,1.37, \\
1.41,1.45,1.49\end{array}$ \\
\hline 4585.48 & 21801.88 & $\left({ }^{3} \mathrm{P}\right) 6 p^{4} \mathrm{D}_{3}^{\circ} 1 / 2-\left({ }^{3} \mathrm{P}\right) 6 d^{4} \mathrm{D}_{31 / 2}$ & $(0.00)$ & 1. 33 & $(0.16) 1.37$ & $\begin{array}{ccc}(0.01, & 0.04, & 0.07, \\
0.10) & 1.28, & 1.31, \\
1.34, & 1.37, & 1.40, \\
1.43, & 1.46\end{array}$ \\
\hline 4592.05 & 21770.69 & (3P) $6 p{ }^{4} \mathrm{~S}_{1 / 2}^{0}-\left({ }^{3} \mathrm{P}\right) 6 d^{4} \mathrm{P}_{2 / 2}$ & & & $(0.00) 1.06$ & $\begin{array}{l}(0.04,0.12) 1.03, \\
1.11,1.19,1.27\end{array}$ \\
\hline 4603. 03 & 21718. 76 & $\left({ }^{3} \mathrm{P}\right) 68^{4} \mathrm{P}_{11 / 2}-\left({ }^{3} \mathrm{P}\right) 6 p^{4} \mathrm{D}_{11 / 2}$ & $(0.00)$ & 1.39 & $(0.00) 1.35$ & $(0.00,0.0 \mathrm{i}) \quad 1.38$, \\
\hline 4615. 50 & 21660.08 & ( $\left.{ }^{1} \mathrm{D}\right) 6 s^{2} \mathrm{D}_{21 / 2}-\left({ }^{1} \mathrm{D}\right) 6 p^{2} \mathrm{P}_{1 / 2}^{\circ}$ & $(0.0$ & 5 & $(0.00) 1.04$ & $\begin{array}{l}(0.07,0.20) \quad 1.05, \\
1.18,1.31,1.44\end{array}$ \\
\hline $\begin{array}{l}4633.30 \\
4651.94\end{array}$ & $\begin{array}{l}21576.87 \\
21490.41\end{array}$ & $\begin{array}{l}\left.{ }^{3} \mathrm{P}\right) 5 d^{4} \mathrm{~F}_{21 / 2}-\left({ }^{3} \mathrm{P}\right) 6 p^{2} \mathrm{D}_{21 / 2}^{2} \\
\text { (3P) } 68^{2}{ }^{2} \mathrm{P}_{11 / 2}-\left({ }^{3} \mathrm{P}\right) 6 p^{2} \mathrm{D}_{11 / 2}^{1}\end{array}$ & $(\overrightarrow{0.00)}$ & $\begin{array}{l}0.75 \\
1.52\end{array}$ & $(0.39) 1.41$ & $\left(\begin{array}{lrl}0.10, & 0.31) & 0.27,\end{array}\right.$ \\
\hline $\begin{array}{l}4668.49 \\
4674.56\end{array}$ & $\begin{array}{l}21414.23 \\
21386.42\end{array}$ & $\begin{array}{l}\text { (1D) } 5 d^{2} \mathrm{D}_{11 / 2}-\left({ }^{1} \mathrm{D}\right) 6 p^{2} \mathrm{P}_{01 / 2}^{0} \\
\left.\text { ( }{ }^{3} \mathrm{P}\right) 5 d^{4} \mathrm{D}_{21 / 2}-\left({ }^{3} \mathrm{P}\right) 6 p^{4} \mathrm{D}_{13 / 2}^{0}\end{array}$ & $(-H)$ & & $(0.26) 0.98,1.50$ & $\begin{array}{l}(0.26) 0.98,1.50 \\
(0.02,0.06) 1.28, \\
132,1.36,1.40\end{array}$ \\
\hline 4698. 01 & 21279.67 & ( $\left.{ }^{3} \mathrm{P}\right) 6 p^{4} \mathrm{D}_{1 / 2}^{\mathrm{i}}-12_{11 / 2}$ & $(\longrightarrow)$ & 1.18 & & $\begin{array}{cc}(0.20, & 0.59) \\
1.18,1.57 & 0.79,\end{array}$ \\
\hline $\begin{array}{l}4712.63 \\
4769.05\end{array}$ & $\begin{array}{l}21213.66 \\
20962.69\end{array}$ & ( $\left.{ }^{3} \mathrm{P}\right) 5 d^{2} \mathrm{D}_{11 / 2}-\left({ }^{1} \mathrm{D}\right) 6 p^{2} \mathrm{~F}_{21 / 2}^{0}$ & $(\overrightarrow{-H})$ & & & 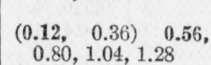 \\
\hline $\begin{array}{l}4779.18 \\
4787.77\end{array}$ & $\begin{array}{l}20918.26 \\
20880.73\end{array}$ & $\begin{array}{r}s p^{6}{ }^{2} \mathrm{~S}_{01 / 2}-\left({ }^{3} \mathrm{P}\right) 6 p^{4} \mathrm{P}_{11 / 2}^{0} \\
\text { (1D) } 5 d^{2} \mathrm{D}_{11 / 2}-\left({ }^{1} \mathrm{D}\right) 6 p^{2} \mathrm{D}_{21 / 2}^{1}\end{array}$ & $(\overrightarrow{0.00)})$ & $\begin{array}{l}1.48 \\
1.20\end{array}$ & & $\begin{array}{l}(0.19) 1.43,1.81 \\
(0.01,0.03) \quad 1.19, \\
1.21,1.23,1.25\end{array}$ \\
\hline 4818. 02 & 20749. 63 & $\left({ }^{3} \mathrm{P}\right) 5 d^{4} \mathrm{D}_{11 / 3}-\left({ }^{3} \mathrm{P}\right) 6 p^{4} \mathrm{D}^{\mathrm{i}} 1 / 2$ & $(-)$ & 0 & & $\begin{array}{ccc}(0.10, & 0.31) & 1.07 \text {, } \\
1.28,1.49 & \end{array}$ \\
\hline $\begin{array}{l}4823.35 \\
4823.41\end{array}$ & $\left.\begin{array}{l}20726.70 \\
20726.45\end{array}\right\}$ & ( $\left.{ }^{3} \mathrm{P}\right) 6 p{ }^{4} \mathrm{P}_{11 / 2}^{0}-\left({ }^{3} \mathrm{P}\right) 7 s^{4} \mathrm{P}_{21 / 2}$ & $(-)$ & 1. $39 H$ & & $\left\{\begin{array}{c}0.04,0.13) 1.39, \\
1.48,1.57,1.66\end{array}\right.$ \\
\hline 4853. 77 & 20596.81 & $\begin{array}{l}\left({ }^{3} \mathrm{P}\right) 6 s^{4} \mathrm{P}_{21 / 2}-\left({ }^{3} \mathrm{P}\right) 6 p{ }^{4} \mathrm{D}_{31 / 2}^{0} \\
\left({ }^{1} \mathrm{D}\right) 5 d^{2} \mathrm{D}_{11 / 2}-\left({ }^{1} \mathrm{D}\right) 6 p^{2} \mathrm{D}_{1 / 2}^{\circ}\end{array}$ & $(-)$ & 1.23 & $(0.00) 1.04$ & $\begin{array}{ccc}(0.08, & 0.25, & 0.42) \\
0.97 & 1.14, & 1.31, \\
1.48,1.65, & 1.82 \\
(0.15,0.44) & 0.81, \\
1.10,1.39 & \end{array}$ \\
\hline $\begin{array}{l}4862.45 \\
4862.54\end{array}$ & $\left.\begin{array}{l}20560.04\} \\
20559.66\end{array}\right\}$ & $\left.{ }^{3} \mathrm{P}\right) 6 p^{4} \mathrm{P}_{21 / 2}^{0}-\left({ }^{3} \mathrm{P}\right) 7 s^{4} \mathrm{P}_{2 / 2}$ & $(-$ & 3 & $(0.00) 1.50$ & $\left\{\begin{array}{ccc}(0.02, & 0.07, & 0.12) \\
1.41, & 1.46, & 1.51, \\
1.56,1.61 & \end{array}\right.$ \\
\hline 4876.50 & 20500.80 & $\left({ }^{3} \mathrm{P}\right) 5 d^{2} \mathrm{D}_{21 / 2}-\left({ }^{1} \mathrm{D}\right) 6 p^{2} \mathrm{~F}_{3}^{\circ} 1 / 2$ & $(0.00)$ & 0.96 & $(0.00) 0.89$ & 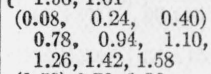 \\
\hline 4883.53 & 20 & ${ }^{(3 \mathrm{P}}$ & $(0.58) 0$ & & $(-\rightarrow) 0$ & $(0.58) 0.70,1.86$ \\
\hline & & & & & $(0.37) 144$ & $\begin{array}{l}(0.10,0.29) \\
1.49,1.68\end{array}$ \\
\hline 4890. 09 & 20443.83 & $\left({ }^{3} \mathrm{P}\right) 6 s^{4} \mathrm{P}_{23 / 2}-\left({ }^{3} \mathrm{P}\right) 6 p{ }^{4} \mathrm{D}_{21 / 2}^{0}$ & $(0.76)$ & 1. $40 \mathrm{H}$ & & 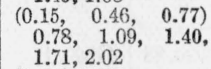 \\
\hline $\begin{array}{l}4919.66 \\
4921.48\end{array}$ & $\begin{array}{l}20320.95 \\
20313.44\end{array}$ & $\begin{array}{l}\left({ }^{3} \mathrm{P}\right) 6 s^{2} \mathrm{P}_{01 / 5}-\left({ }^{3} \mathrm{P}\right) 6 p^{2} \mathrm{P}_{01 / 2}^{0} \\
\left.{ }^{3} \mathrm{P}\right) 6 s^{2} \mathrm{P}_{11 / 5}-\left({ }^{3} \mathrm{P}\right) 6 p^{2} \mathrm{D}_{21 / 2}^{1}\end{array}$ & $(-\overline{0.00)}$ & $\begin{array}{l}0.88 \\
1.25\end{array}$ & $(0.00) 1.21$ & $\begin{array}{l}(0.28) \\
(0.08,0.84 \\
1.32,1.49,1.66\end{array}$ \\
\hline 4972. 71 & 20104.17 & $\left({ }^{3} \mathrm{P}\right) 5 d^{3} \mathrm{D}_{23 / 2}-\left({ }^{1} \mathrm{D}\right) 6 p^{2} \mathrm{P}_{11 / 2}$ & $(0.00)$ & 1.30 & $(0.00) \cdot .23$ & $\begin{array}{c}(0.01,0.04) 1.29 \text {, } \\
1.32,1.35,1.38\end{array}$ \\
\hline
\end{tabular}


TABLE 1.-Zeeman patterns in Xe II-Continued

\begin{tabular}{|c|c|c|c|c|c|}
\hline $\begin{array}{c}\text { Wave } \\
\text { length } \\
\text { (air) }\end{array}$ & $\begin{array}{l}\text { Wave } \\
\text { number } \\
(\mathrm{vac})\end{array}$ & Term combination & $\begin{array}{c}\text { Observed } \\
\text { Zeeman pattern } \\
\text { Humphreys- } \\
\text { Meggers }\end{array}$ & $\begin{array}{c}\text { Observed } \\
\text { Zeeman pattern } \\
\text { deBruin }\end{array}$ & $\begin{array}{c}\text { Calculated Zeeman } \\
\text { pattern }\end{array}$ \\
\hline $\begin{array}{c}A \\
4988.77 \\
5044.92 \\
5080.62\end{array}$ & $\begin{array}{c}c m^{-1} \\
20039.45 \\
19816.41 \\
19677.17\end{array}$ & 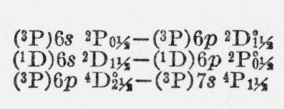 & $(\longrightarrow) 1.72$ & $\begin{array}{l}(0.09) 1.02 \\
(0.00) 1.10\end{array}$ & $\begin{array}{l}(0.40) 0.96,1.77 \\
(0.13) 0.84,1.10 \\
(0.10,0.30) \quad 0.94,\end{array}$ \\
\hline $\begin{array}{l}5191.37 \\
5260.44 \\
5261.95\end{array}$ & $\begin{array}{l}19257.39 \\
19004.54 \\
18999.09\end{array}$ & 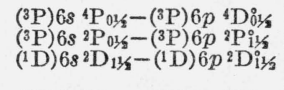 & $\begin{array}{l}(0.82) 1.45 \\
(0.00) 1.76 \\
(0.00) 0.94\end{array}$ & $\begin{array}{l}(0.86) 1.43 \\
(0.35) 1.55 \\
(0.07) 0.92\end{array}$ & $\begin{array}{l}(0.91) 1.54,1.81 \\
(0.42) 0.98,1.81 \\
(0.01,0.04) \quad 0.91,\end{array}$ \\
\hline 5292.22 & 18890.42 & $\left({ }^{3} \mathrm{P}\right) 6 s^{4} \mathrm{P}_{23,5}-\left({ }^{3} \mathrm{P}\right) 6 p{ }^{4} \mathrm{P}_{23 / 5}^{0}$ & $(0.00) 1.52$ & $(0.20) 1.48$ & $\begin{array}{ccc}(0.04, & 0.12, & 0.20) \\
1.36, & 1.44, & 1.52, \\
1.60,1.68 & \end{array}$ \\
\hline 5309.27 & 18829.76 & $\left({ }^{3} \mathrm{P}\right) 6 \varepsilon^{2} \mathrm{P}_{1 / 5}-\left({ }^{3} \mathrm{P}\right) 6 p{ }^{4} \mathrm{Si}^{\mathrm{N} / 5}$ & $(0.41) 1.42$ & & $\begin{array}{cc}(0.15,0.45) & 1.13, \\
1.43,1.73 & \end{array}$ \\
\hline 5313.87 & 18813.46 & $\left({ }^{3} \mathrm{P}\right) 6 p{ }^{4} \mathrm{D}_{31 / 3}^{8}-\left({ }^{3} \mathrm{P}\right) 7 s^{4} \mathrm{P}_{21 / 3}$ & & $(0.00) 0.80$ & $\begin{array}{ccc}(0.07, & 0.21, & 0.35) \\
1.04, & 1.18, & 1.32, \\
1.46, & 1.60, & 1.74\end{array}$ \\
\hline 5339.35 & 18723.67 & $\left({ }^{3} \mathrm{P}\right) 6 s^{4} \mathrm{P}_{21 / 2}-\left({ }^{3} \mathrm{P}\right) 6 p{ }^{4} \mathrm{P}_{1 / 5}^{0}$ & $(0.00) 1.52$ & $(0.00) 1.50$ & $\begin{array}{l}(0.03,0.09) 1.47, \\
1.53,1.59,1.65\end{array}$ \\
\hline $\begin{array}{l}5372.39 \\
5419.15\end{array}$ & $\begin{array}{l}18608.53 \\
18447.96\end{array}$ & 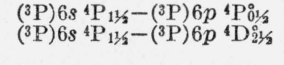 & $\begin{array}{l}(0.00) 1.37 \\
(0.00) 1.14\end{array}$ & $\begin{array}{l}(0.00) 1.32 \\
(0.00) 1.06\end{array}$ & $\begin{array}{l}(0.06) 1.33,1.45 \\
(0.07,0.21) 1.04, \\
1.18,1.32,1.46\end{array}$ \\
\hline $\begin{array}{l}5438.96 \\
5460.39\end{array}$ & $\begin{array}{l}18380.77 \\
18308.63\end{array}$ & 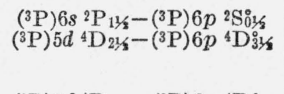 & $\begin{array}{l}(0.24) 1.32,1.80 \\
(0.00) 1.45\end{array}$ & $(0.27) 1.25$ & $\begin{array}{l}(0.24) 1.34,1.82 \\
(0.02,0.07,0.12) \\
1.26,1.31,1.36 \text {, } \\
1.41,1.46,1.51\end{array}$ \\
\hline 5472.61 & 18267.75 & $\left({ }^{3} \mathrm{P}\right) 5 d^{4} \mathrm{D}_{31 / 2}-\left({ }^{3} \mathrm{P}\right) 6 p{ }^{4} \mathrm{D}_{3}{ }^{3} / 6$ & $(0.00) 1.39$ & & $\begin{array}{ccc}(0.00, & 0.01, & 0.02 \\
0.03) & 1.36, & 1.37 \\
1.38, & 1.39, & 1.40 \\
1.41, & 1.42\end{array}$ \\
\hline 5531.07 & 18074.68 & $\left({ }^{3} \mathrm{P}\right) 5 d^{4} \mathrm{D}_{31 / 5}-\left({ }^{3} \mathrm{P}\right) 6 p{ }^{4} \mathrm{D}_{21 / 3}^{8}$ & $(0.00) 1.60$ & & $\begin{array}{l}(0.07, \quad 0.22, \quad 0.37) \\
1.02,1.17,1.32, \\
1.47,1.62,1.77\end{array}$ \\
\hline $\begin{array}{l}5581.93 \\
5616.67\end{array}$ & $\begin{array}{l}17909.99 \\
17799.21\end{array}$ & ( $\left.{ }^{3} \mathrm{P}\right) 5 d{ }^{2} \mathrm{P}_{136}-\left({ }^{3} \mathrm{P}\right) 6 p^{2} \mathrm{D}_{216}{ }^{2}$ & $(0.00) 1.54$ & $(0.15) 1.00$ & $(0.10,0.31){ }_{1.31,1.10,}^{1.73}$ \\
\hline $\begin{array}{l}5659.38 \\
5667.56 \\
5719.61\end{array}$ & $\begin{array}{l}17664.89 \\
17639.39 \\
17478.87\end{array}$ & 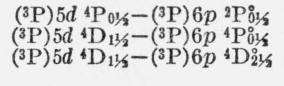 & $\begin{array}{l}(0.36) 1.46 \\
(0.15) 1.05 \\
(0.00) 1.32\end{array}$ & & $\begin{array}{l}(0.36) 1.46 \\
(0.17) 1.00,1.34 \\
(0.04,0.12) 1.13,\end{array}$ \\
\hline 5726.91 & 17456.59 & ( $\left.{ }^{1} \mathrm{D}\right) 5 d^{2} \mathrm{~F}_{21 / 3}-\left({ }^{1} \mathrm{D}\right) 6 p^{2} \mathrm{D}: 21 / 5$ & $(0.00) 1.15$ & & $\begin{array}{l}(0.07,0.21,0.35) \\
0.87,1.01,1.15, \\
1.29,1.43\end{array}$ \\
\hline $\begin{array}{l}5751.03 \\
5758.65\end{array}$ & $\begin{array}{l}17383.38 \\
17360.38\end{array}$ & 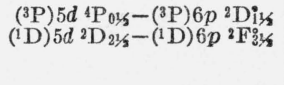 & $\begin{array}{l}(0.00) 1.22 \\
(0.00) 1.27\end{array}$ & & $\begin{array}{l}(0.22) 1.15,1.60 \\
(0.03,0.10, \quad 0.17) \\
1.00,1.07,1.14,\end{array}$ \\
\hline 5776. 39 & 17307.06 & ( $\left.{ }^{3} \mathrm{P}\right) 5 d{ }^{2} \mathrm{P} 01 / 2-\left({ }^{3} \mathrm{P}\right) 6 p{ }^{2} \mathrm{P}_{13 / 3}$ & $(0.00) 1.33$ & & $(0.01) 1.39$ \\
\hline $\begin{array}{l}5905.13 \\
5945.53\end{array}$ & $\begin{array}{l}16929.75 \\
16814.71\end{array}$ & 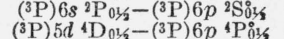 & $\begin{array}{l}(0.77) 1.31 \\
(0.46) 0.96\end{array}$ & & $\begin{array}{l}(0.75) 1.31 \\
(0.50) \\
1.00\end{array}$ \\
\hline 5976.46 & 16727.69 & ( $\left.{ }^{3} \mathrm{P}\right) 6 s^{4} \mathrm{P}_{11 / 3}-\left({ }^{3} \mathrm{P}\right) 6 p{ }^{4} \mathrm{P}_{1 / 3}^{01 / 3}$ & $(0.30) 1.50$ & & $\begin{array}{ll}(0.11,0.34) & 1.27, \\
1.50,1.73 & \end{array}$ \\
\hline 6036.20 & 16562.14 & $\left({ }^{3} \mathrm{P}\right) 5 d^{4} \mathrm{D}_{21 / 5}-\left({ }^{3} \mathrm{P}\right) 6 p{ }^{4} \mathrm{P}_{21 / 3}^{0}$ & $(0.27) 1.41$ & & 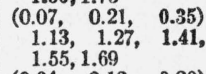 \\
\hline 6051.15 & 16521. 22 & $\left({ }^{3} \mathrm{P}\right) 5 d^{4} \mathrm{D}_{31 / 2}-\left({ }^{3} \mathrm{P}\right) 6 p{ }^{4} \mathrm{P}_{21 / 2}^{0}$ & $(0.00) 1.29$ & & $\begin{array}{l}(0.04,0.12, \quad 0.20) \\
1.20,1.28,1.36, \\
1.44,1.52,1.60\end{array}$ \\
\hline 6097.59 & 16395.39 & $\left({ }^{3} \mathrm{P}\right) 5 d^{4} \mathrm{D}_{23 / 2}-\left({ }^{3} \mathrm{P}\right) 6 p{ }^{4} \mathrm{P}_{1 / 3}{ }^{1}$ & $(0.37) 1.07$ & & $(0.14, \quad 0.42) \quad 0.92$, \\
\hline 6101.43 & 16385.07 & ( $\left.{ }^{3} \mathrm{P}\right) 5 d^{2} \mathrm{D}_{11 / 3}-\left({ }^{3} \mathrm{P}\right) 6 p^{2} \mathrm{D}_{136}$ & $(0.00) 1.26$ & & $\begin{array}{ll}(0.10,0.31) & 1.05 \\
1.26,1.47 & \end{array}$ \\
\hline $\begin{array}{l}6270.82 \\
6277.54\end{array}$ & $\begin{array}{l}15942.48 \\
15925.41\end{array}$ & $\begin{array}{l}\text { (1D) } 6 s^{2} \mathrm{D}_{1 / 3}-\left({ }^{1} \mathrm{D}\right) 6 p^{2} \mathrm{~F}_{21 / 5}^{0} \\
\left({ }^{3} \mathrm{P}\right) 5 d^{4} \mathrm{C}_{11 / 5}-\left({ }^{3} \mathrm{P}\right) 6 p^{4} \mathrm{P}^{2}{ }^{21 / 5}\end{array}$ & $\begin{array}{l}(0.00) 0.92 \\
(0.00) 1.70\end{array}$ & & $\begin{array}{l}(0.00) \\
(0.15,0.92 \\
1.32,1.63,1.94\end{array} 1.01$, \\
\hline $\begin{array}{l}6300.86 \\
6343.96\end{array}$ & $\begin{array}{l}15866.47 \\
15758.68\end{array}$ & 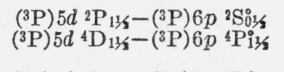 & $\begin{array}{l}(0.51)-\overline{1.00}, 1.57 \\
(0.63)\end{array}$ & & $\begin{array}{l}(0.43) 0.77,1.63 \\
(0.22,0.67) \quad 0.95, \\
1.40,1.85\end{array}$ \\
\hline 6512.83 & 15350.07 & $\left({ }^{3} \mathrm{P}\right) 5 d^{2} \mathrm{D}_{11 / 5}-\left({ }^{3} \mathrm{P}\right) 6 p^{2} \mathrm{P}_{1 / 2}{ }^{1}$ & $(0.00) 1.23$ & & $\begin{array}{l}(0.12,0.35) \quad 1.04, \\
1.27,1.40\end{array}$ \\
\hline $\begin{array}{l}6595.01 \\
6805.74\end{array}$ & $\begin{array}{l}15158.80 \\
14689.43\end{array}$ & $\left({ }^{3} \mathrm{P}\right) 5 d^{4} \mathrm{~F}_{31 / 5}-\left({ }^{3} \mathrm{P}\right) 6 p{ }^{4} \mathrm{D}_{21 / 3}^{\circ}$ & $\begin{array}{l}(0.00) 1.02 \\
(0.00) 0.85\end{array}$ & & 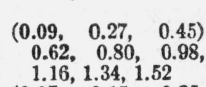 \\
\hline 6990.88 & 14300.41 & $\left({ }^{3} \mathrm{P}\right) 5 d{ }^{4} \mathrm{~F}_{41 / 5}-\left({ }^{3} \mathrm{P}\right) 6 p{ }^{1} \mathrm{D}_{3 / 6}^{\circ}$ & $(0.00) 1.10$ & & $\begin{array}{ccc}(0.05, & 0.15, & 0.25, \\
0.35) & 0.94, & 1.04 \\
1.14, & 1.24, & 1.34, \\
1.44, & 1.54,1.64\end{array}$ \\
\hline
\end{tabular}


As the two sets of data were obtained in different laboratories and by different observers using techniques not exactly the same, it has been decided to make a separate tabulation. In general, reasonably good agreement has been obtained from lines involving levels of low $J$ value which have relatively simple patterns. In a majority of instances both deBruin's displacement of components and $g$ values are somewhat lower than those observed at the NBS. No very plausible reason for the discrepancy has been found. The field strengths in the case of the Washington observations were calculated partly from patterns obtained with metallic spark sources and partly from the sodium lines appearing in the tube discharge. No appreciable discrepancies were found for the resulting field strengths calculated from patterns yielded by these different types of sources when both were operated during the same run of the magnet.

The calculated Zeeman patterns appearing in the final column of table 1 were obtained from the $g$ values derived from the observations by Humphreys and Meggers, where such observed g's were available. The remaining calculated patterns, some 20 in all, were computed from $g$ values derived from deBruin's data. In some cases the $g$ value could be established only from deBruin's observations. The best instance is that of the level ( $\left.{ }^{1} \mathrm{D}\right) 6 p^{2} \mathrm{P}_{01 / 2}^{\circ}$, which was observed by deBruin in several combinations giving well-resolved patterns, whereas the same lines were too weak to appear on our plates with the exposures used.

Observed and calculated $g$ values in Xe II are listed in table 2 with the corresponding level values and designations. In successive columns are shown parentages, electron configurations, quantum designations, and numerical values of the various levels. These are followed in order by the appropriate $g$ values, observed by Humphreys and Meggers, observed by deBruin, calculated by the Landé formula, and calculated for $J j$ coupling. The term table does not list all known levels but is complete as far as the levels due to $6 s, 6 p$, or $5 d$ are concerned. That is, a place is assigned to all such levels in the table, even though some of them are still unknown. This was done in order that complete theoretical calculations might be shown. Only those levels of higher $n$ value are listed for which $g$ values have been determined from observations. A few levels are given a different designation from that indicated in the earlier publication, although all $J$ values have been retained. These changes are discussed in succeeding paragraphs. Previous assignments [2] are listed in the final column of table 2. The theoretical calculation of $g$ 's for $J j$ coupling is correct for the groups as a whole, because it can be verified by the $g$-sum rule. Individual correlations of levels for $L S$ and $J j$ coupling are necessarily somewhat arbitrary, since it is not certain how series-forming terms approach their limits in spectra of noble-gas type. The correlations are based largely on evidence from observed $g$ 's. It is apparent that in a spectrum showing higher series numbers, if successive members of the same series yielded the same observed $g$ values, in other words, if Preston's law were obeyed, these $g$ values might assist in determining how the levels of a multiple limit are approached. This statement applies only to cases where the valence electron is added in $J j$ fashion or very nearly so. 
Table 2.-g values-Xe ir levels

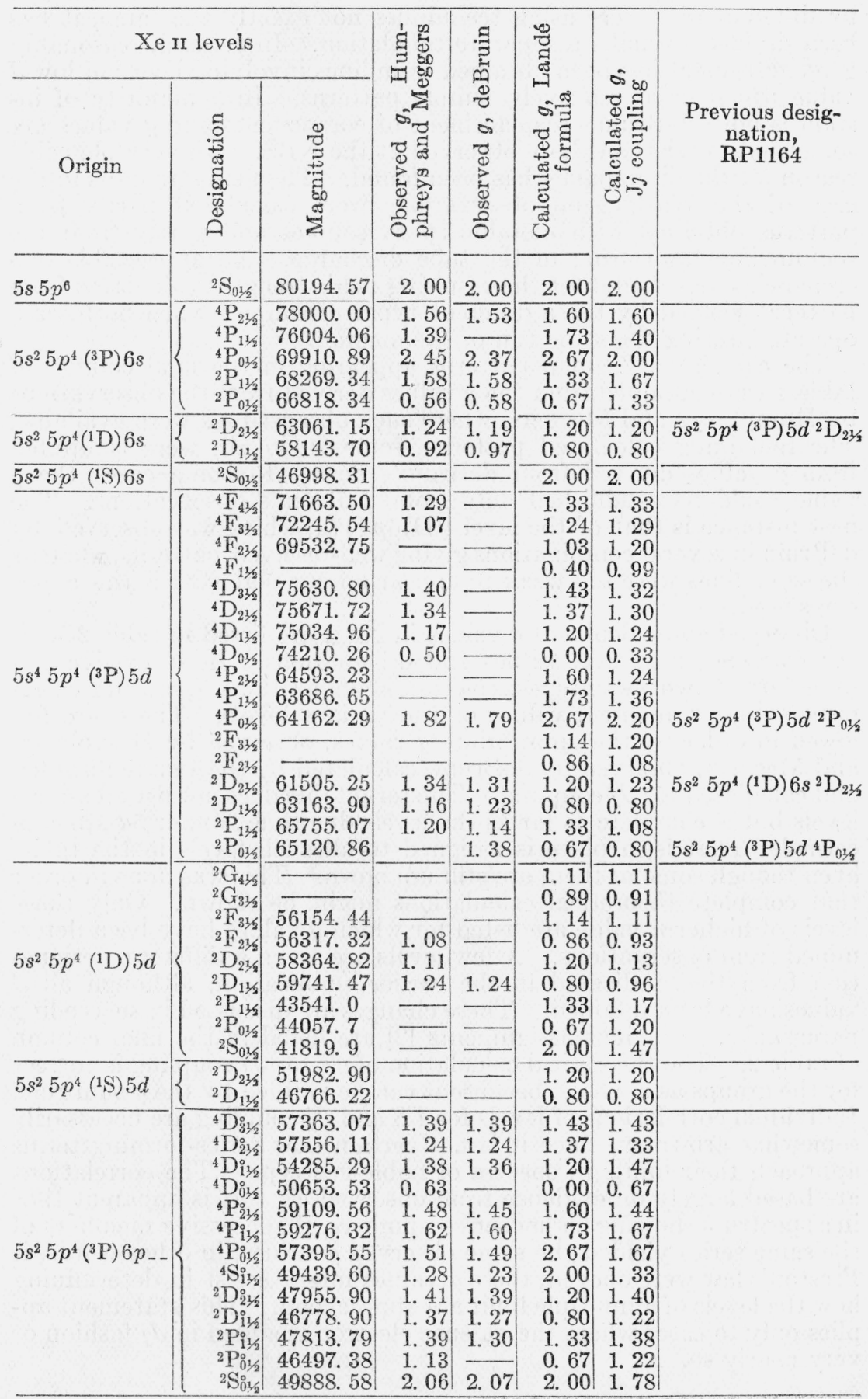


TABLE 2.-g values-Xe Ir levels-Continued

\begin{tabular}{|c|c|c|c|c|c|c|c|}
\hline \multicolumn{3}{|c|}{ Xe II levels } & \multirow{2}{*}{ 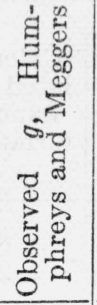 } & \multirow{2}{*}{ 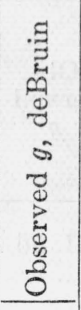 } & \multirow{2}{*}{ 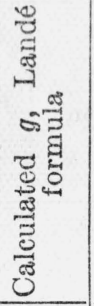 } & \multirow[b]{2}{*}{ 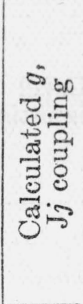 } & \multirow[b]{2}{*}{$\begin{array}{c}\text { Previous desig- } \\
\text { nation, } \\
\text { RP1164 }\end{array}$} \\
\hline Origin & 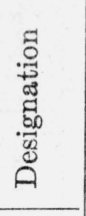 & 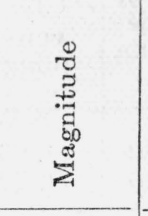 & & & & & \\
\hline $5 s^{2} 5 p^{4}\left({ }^{1} \mathrm{D}\right) 6 p$ & $\begin{array}{l}{ }^{2} \mathrm{~F}_{31 / 2}^{\circ} \\
{ }^{2} \mathrm{~F}_{21 / 2}^{\circ} \\
{ }^{2} \mathrm{D}_{21 / 2}^{\circ} \\
{ }^{2} \mathrm{D}_{11 / 2}^{\circ} \\
{ }^{2} \mathrm{P}_{11 / 2}^{\circ} \\
{ }^{2} \mathrm{P}_{01 / 2}^{\circ} \\
\end{array}$ & $\begin{array}{l}41004.37 \\
42201.21 \\
38860.72 \\
39144.64 \\
41401.03 \\
38327.31 \\
\end{array}$ & $\begin{array}{l}1.18 \\
0.92 \\
1.22 \\
0.95 \\
1.37 \\
\end{array}$ & \begin{tabular}{l|}
$\frac{1.13}{1.17}$ \\
1.40 \\
0.71 \\
\end{tabular} & $\begin{array}{l}1.14 \\
0.86 \\
1.20 \\
0.80 \\
1.33 \\
0.67 \\
\end{array}$ & \begin{tabular}{l|}
1.14 \\
0.93 \\
1.12 \\
1.07 \\
1.07 \\
0.67 \\
\end{tabular} & \\
\hline $5 s^{2} 5 p^{4}\left({ }^{1} \mathrm{~S}\right) 6 p \ldots$ & $\begin{array}{l}{ }^{2} \mathrm{P}_{1 / 2}^{0} \\
{ }^{2} \mathrm{P}_{01 / 5}^{0}\end{array}$ & 30858.18 & & & $\begin{array}{l}1.33 \\
0.67\end{array}$ & \begin{tabular}{l|}
1.33 \\
0.67
\end{tabular} & \\
\hline $5 s^{2} 5 p^{4}\left({ }^{3} \mathrm{P}\right) 7 s_{-}$ & $\begin{array}{l}{ }^{4} \mathrm{P}_{21 / 2} \\
{ }^{4} \mathrm{P}_{1 / 1} \\
{ }^{4} \mathrm{P}_{01 / 2}\end{array}$ & $\begin{array}{l}38549.7 \\
37879.2 \\
36007.5 \\
\end{array}$ & $\frac{1.53}{2.06}$ & $\begin{array}{l}1.52 \\
1.45 \\
1.98 \\
\end{array}$ & $\begin{array}{l}\text { 1. } 60 \\
\text { 1. } 73 \\
2.67\end{array}$ & $\begin{array}{l}1.60 \\
\text { 1. } 40 \\
\text { 2. } 00 \\
\end{array}$ & \\
\hline $5 s^{2} 5 p^{4}\left({ }^{1} \mathrm{D}\right) 7 s_{--}$ & $\begin{array}{l}{ }^{2} \mathrm{D}_{21 / 2} \\
{ }^{2} \mathrm{D}_{11 / 2}\end{array}$ & $\begin{array}{l}24763.0 \\
21266.0 \\
\end{array}$ & 1.30 & 1.50 & $\begin{array}{l}1.20 \\
0.80 \\
\end{array}$ & $\begin{array}{l}1.20 \\
0.80 \\
\end{array}$ & \\
\hline $5 s^{2} 5 p^{4}\left({ }^{3} \mathrm{P}\right) 6 d_{--}$ & $\begin{array}{l}{ }^{4} \mathrm{~F}_{41 / 2} \\
{ }^{4} \mathrm{~F}_{31 / 3} \\
{ }^{4} \mathrm{~F}_{21 / 2} \\
{ }^{4} \mathrm{~F}_{11 / 2} \\
{ }^{4} \mathrm{D}_{31 / 2} \\
{ }^{4} \mathrm{D}_{21 / 2} \\
{ }^{4} \mathrm{D}_{11 / 2} \\
{ }^{4} \mathrm{D}_{01 / 2} \\
{ }^{4} \mathrm{P}_{21 / 2} \\
{ }^{4} \mathrm{P}_{11 / 2} \\
{ }^{4} \mathrm{P}_{01 / 2} \\
{ }^{2} \mathrm{D}_{21 / 2} \\
{ }^{2} \mathrm{D}_{11 / 2} \\
{ }^{2} \mathrm{P}_{11 / 3} \\
{ }^{2} \mathrm{P}_{01 / 2}\end{array}$ & $\begin{array}{r}34470.7 \\
31974.3 \\
35561.2 \\
35521.4 \\
35360.2 \\
27669.0 \\
26928.2 \\
30185.0 \\
26683.4 \\
25128.3 \\
25845.7\end{array}$ & $\begin{array}{l}1.13 \\
1.36 \\
1.43 \\
1.64 \\
\square \\
0.98 \\
\square \\
\\
\end{array}$ & \begin{tabular}{l|}
1.10 \\
1.13 \\
1.34 \\
1.26 \\
1.35 \\
1.15 \\
0.78 \\
1.19 \\
1.00 \\
0.38 \\
\end{tabular} & $\begin{array}{l}1.33 \\
1.24 \\
1.03 \\
0.40 \\
\text { 1. } 43 \\
\text { 1. } 37 \\
\text { 1. } 20 \\
0.00 \\
1.60 \\
1.73 \\
\text { 2. } 67 \\
\text { 1. } 20 \\
\text { 0. } 80 \\
\text { 1. } 33 \\
\text { 0. } 67\end{array}$ & $\begin{array}{l}1.33 \\
1.29 \\
1.20 \\
0.99 \\
1.32 \\
1.30 \\
1.24 \\
0.33 \\
1.24 \\
1.36 \\
2.20 \\
1.23 \\
0.80 \\
1.08 \\
0.80\end{array}$ & \\
\hline $5 s^{2} 5 p^{4}\left({ }^{1} \mathrm{D}\right) 6 d_{-}$ & $\begin{array}{l}2 D_{21 / 2} \\
{ }^{2} D_{11 / 2}\end{array}$ & $\begin{array}{l}21371.1 \\
22983.2 \\
\end{array}$ & - & $\overline{1.07}$ & $\begin{array}{l}1.20 \\
0.80\end{array}$ & $\begin{array}{l}1.13 \\
0.96\end{array}$ & \\
\hline & $\begin{array}{l}12_{11 / 2} \\
14_{21 / 2} \\
16_{11 / 2} \\
18_{01 / 2} \\
20_{01 / 2} \\
43_{31 / 2}^{\circ} \\
15_{21 / 2}^{\circ}\end{array}$ & $\begin{array}{l}33005.6 \\
24140.6 \\
34559.15 \\
34514.23 \\
32342.0 \\
\\
33574.65 \\
31038.29\end{array}$ & $\underline{0.99}$ & \begin{tabular}{l|}
1.02 \\
0.98 \\
1.68 \\
0.94 \\
1.89 \\
1.06 \\
1.08
\end{tabular} & & & $5 s^{2} 5 p^{4}\left({ }^{3} \mathrm{P}\right) 6 d^{4} \mathrm{D}_{01 / 2}$ \\
\hline
\end{tabular}

Table 3 shows the application of the $g$-sum rule to the $6 s$ and $6 p$ levels of Xe II. The essentially satisfactory manner in which the rule is obeyed, in spite of the fact that a high degree of precision cannot be claimed for our measurements, confirms the assignments of quantum numbers and electron configurations indicated in the previous publication [2]. In cases where only a single observed $g$ is needed to complete the sum, a value is given, in parentheses, which will satisfy the rule. Differences of one or two units in the last place in the sums of theoretical $g$ 's are due to rounding off the $g$ 's to two places of decimals. In the final columns are listed the values of the quantities substituted in the formula for $g$ in case of $J j$ coupling. 
TABLE 3.-Application of the g-sum rule in Xe II

\begin{tabular}{|c|c|c|c|c|c|c|c|c|}
\hline $\begin{array}{l}\text { Configura- } \\
\text { tion }\end{array}$ & $\begin{array}{c}\text { Level } \\
\text { designation }\end{array}$ & $\begin{array}{l}\text { Ob- } \\
\text { served } \\
g\end{array}$ & $\begin{array}{l}\text { Calcu- } \\
\text { lated g, } \\
\text { Landé } \\
\text { formula }\end{array}$ & $\begin{array}{c}\text { Calcu- } \\
\text { lated } g \text {, } \\
J j \\
\text { cou- } \\
\text { pling }\end{array}$ & $J_{p}$ & $j_{i}$ & $g_{\nu}$ & $g_{8}$ \\
\hline \multirow[t]{6}{*}{$5 s^{2} 5 p^{4} 6 s$} & \multirow[t]{3}{*}{$\begin{array}{l}\left({ }^{3} \mathrm{P}\right) \\
{ }^{4}{ }^{4} \mathrm{P}_{21 / 3} \\
\left.{ }^{1}\right)^{2} \mathrm{D}_{21 / 3}\end{array}$} & $\begin{array}{l}\text { 1. } 56 \\
\text { 1. } 24\end{array}$ & $\begin{array}{l}\text { 1. } 60 \\
\text { 1. } 20\end{array}$ & $\begin{array}{l}\text { 1. } 60 \\
\text { 1. } 20\end{array}$ & \multirow[t]{2}{*}{$\begin{array}{l}2 \\
2\end{array}$} & \multirow[t]{2}{*}{$\begin{array}{l}1 / 2 \\
1 / 2\end{array}$} & \multirow[t]{2}{*}{$1^{3 / 4}$} & \multirow[t]{2}{*}{$\begin{array}{l}2 \\
2\end{array}$} \\
\hline & & 2. 80 & 2. 80 & 2. 80 & & & & \\
\hline & & $\begin{array}{l}1.39 \\
\text { 1. } 58 \\
0.92\end{array}$ & $\begin{array}{l}\text { 1. } 73 \\
1.33 \\
0.80\end{array}$ & $\begin{array}{l}\text { 1. } 40 \\
\text { 1. } 67 \\
0.80\end{array}$ & $\begin{array}{l}2 \\
1 \\
2\end{array}$ & $\begin{array}{l}1 / 2 \\
1 / 2 \\
1 / 2\end{array}$ & $\begin{array}{r}3 / 2 \\
3 / 2 \\
1^{3 / 2}\end{array}$ & $\begin{array}{l}2 \\
2 \\
2\end{array}$ \\
\hline & Sum & 3. 89 & 3. 86 & 3. 87 & \multirow{3}{*}{$\begin{array}{l}1 \\
0 \\
0\end{array}$} & \multirow{3}{*}{$\begin{array}{l}1 / 2 \\
1 / 2 \\
1 / 2\end{array}$} & \multirow{3}{*}{$1^{\frac{3 / 2}{\%}}$} & \multirow{3}{*}{$\begin{array}{l}2 \\
2 \\
2\end{array}$} \\
\hline & \multirow[t]{2}{*}{ 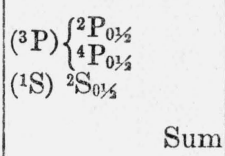 } & $\begin{array}{l}0.56 \\
2.45 \\
(2.32)\end{array}$ & $\begin{array}{l}0.67 \\
2.67 \\
2.00\end{array}$ & $\begin{array}{l}\text { 1. } 33 \\
2.00 \\
2.00\end{array}$ & & & & \\
\hline & & (5. 33) & 5. 34 & 5. 33 & & & & \\
\hline \multirow[t]{8}{*}{$\overline{5 s^{2} 5 p^{4} 6 p}$} & \multirow[b]{3}{*}{ 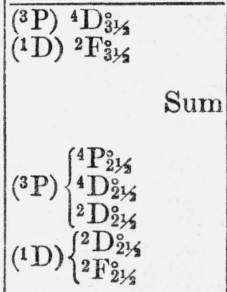 } & $\begin{array}{l}1.39 \\
1.18\end{array}$ & $\begin{array}{l}\text { 1. } 43 \\
\text { 1. } 14\end{array}$ & $\begin{array}{l}\text { 1. } 43 \\
1.14\end{array}$ & \multirow[t]{2}{*}{$\begin{array}{l}2 \\
2\end{array}$} & \multirow[t]{2}{*}{$\begin{array}{l}3 / 2 \\
3 / 2\end{array}$} & \multirow[t]{2}{*}{$1^{3 / 2}$} & \multirow[t]{2}{*}{$\begin{array}{l}4 / 3 \\
4 / 3\end{array}$} \\
\hline & & 2. 57 & 2. 57 & 2. 57 & & & & \\
\hline & & $\begin{array}{l}\text { 1. } 48 \\
1.24 \\
1.41 \\
1.22 \\
0.92\end{array}$ & $\begin{array}{l}\text { 1. } 60 \\
\text { 1. } 37 \\
\text { 1. } 20 \\
\text { 1. } 20 \\
\text { 0. } 86\end{array}$ & $\begin{array}{l}\text { 1. } 44 \\
1.33 \\
1.40 \\
1.12 \\
0.93\end{array}$ & $\begin{array}{l}2 \\
2 \\
1 \\
2 \\
2\end{array}$ & $\begin{array}{l}3 / 2 \\
1 / 2 \\
3 / 2 \\
3 / 2 \\
1 / 2\end{array}$ & $\begin{array}{l}3 / 2 \\
3 / 2 \\
3 / 2 \\
1^{3 / 2} \\
1\end{array}$ & $\begin{array}{l}4 / 3 \\
2 / 3 \\
4 / 3 \\
4 / 3 \\
2 / 3\end{array}$ \\
\hline & Sum & 6. 27 & 6. 23 & 6. 22 & \multirow[b]{2}{*}{\begin{tabular}{l|}
2 \\
2 \\
1 \\
1 \\
0 \\
2 \\
2 \\
0
\end{tabular}} & \multirow[b]{2}{*}{$\begin{array}{l}3 / 2 \\
1 / 2 \\
3 / 2 \\
1 / 2 \\
3 / 2 \\
3 / 2 \\
1 / 2 \\
3 / 2\end{array}$} & \multirow[b]{2}{*}{$\begin{array}{l}3 / 2 \\
3 / 2 \\
3 / 2 \\
3 / 2 \\
0 \\
1 \\
1 \\
1\end{array}$} & \multirow[b]{2}{*}{$\begin{array}{l}4 / 3 \\
2 / 3 \\
4 / 3 \\
2 / 3 \\
4 / 3 \\
4 / 3 \\
2 / 3 \\
4 / 3\end{array}$} \\
\hline & 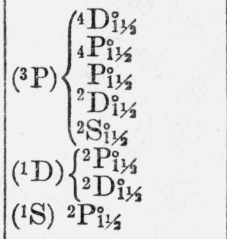 & $\begin{array}{l}1.38 \\
1.62 \\
1.39 \\
1.37 \\
1.28 \\
1.37 \\
0.95 \\
(1.17)\end{array}$ & $\begin{array}{l}1.20 \\
1.73 \\
\text { 1. } 33 \\
0.80 \\
2.00 \\
1.33 \\
0.80 \\
1.33\end{array}$ & $\begin{array}{l}1.47 \\
1.67 \\
1.38 \\
1.22 \\
1.33 \\
1.07 \\
1.07 \\
1.33\end{array}$ & & & & \\
\hline & (1S) ${ }^{2} \mathrm{P}_{11 / 3}^{0}$ & (10. 53) & 10. 52 & 10. 54 & \multirow{3}{*}{$\begin{array}{l}2 \\
1 \\
1 \\
0 \\
2 \\
0\end{array}$} & \multirow{3}{*}{$\begin{array}{l}3 / 2 \\
3 / 2 \\
1 / 2 \\
1 / 2 \\
3 / 2 \\
1 / 2 \\
1 / 2\end{array}$} & \multirow{3}{*}{\begin{tabular}{l|}
32 \\
$3 / 2$ \\
$3 / 2$ \\
$3 \%$ \\
$\%$ \\
$1^{\%}$ \\
1
\end{tabular}} & \multirow{3}{*}{$\begin{array}{l}4 / 3 \\
4 / 3 \\
2 / 3 \\
2 / 3 \\
4 / 3 \\
2 / 3\end{array}$} \\
\hline & \multirow[t]{2}{*}{$\begin{array}{l}\left.\text { ( }{ }^{3} \mathrm{P}\right)\left\{\begin{array}{l}{ }^{4} \mathrm{P}_{01 / 2}^{0} \\
{ }^{2} \mathrm{P}_{01 / 2} \\
{ }^{2} \mathrm{~S}_{01 / 2}^{0} \\
{ }^{4} \mathrm{D}_{01 / 2}^{0}\end{array}\right. \\
\left({ }^{1} \mathrm{D}\right){ }^{2} \mathrm{P}_{01 / 3}^{0} \\
\text { (1S) }{ }^{2} \mathrm{P}_{01 / 3}^{0}\end{array}$} & $\begin{array}{l}1.51 \\
1.13 \\
2.06 \\
0.63 \\
0.71 \\
(0.64)\end{array}$ & $\begin{array}{l}2.67 \\
0.67 \\
2.00 \\
0.00 \\
0.67 \\
0.67\end{array}$ & $\begin{array}{l}1.67 \\
1.22 \\
1.78 \\
0.67 \\
0.67 \\
0.67\end{array}$ & & & & \\
\hline & & (0.68) & 6. 68 & 6. 68 & & & & \\
\hline
\end{tabular}

Application of the $g$-sum rule has shown the need of a few changes of assignment of quantum numbers. The designations of levels 61505.25 and 63061.15 are interchanged, also 64162.29 and 65120.86 . In the earlier work there was no basis for distinguishing such levels, which showed similar combining properties. 
The level 32342.0, previously designated $\left({ }^{3} \mathrm{P}\right) 6 d{ }^{4} \mathrm{D}_{01 / 1}$, has a $g$ value, 1.89 , which makes that designation untenable. In the present paper, it is simply assigned a number, $20_{015}$.

Tables 1 and 2 incorporate the revisions in classifications, resulting from new designations of certain levels, which are required to bring the corresponding tables of RP1164 [2] up to date, to the extent permitted by the limitation of table 1 to the list of lines for which Zeeman patterns have been observed. These changes will necessarily affect the classifications of a number of other lines, involving newly designated levels, for which Zeeman effects have not been observed.

Zeeman patterns have been observed for several unclassified lines. The line at $4037.29 \mathrm{~A}$ exhibits a sextet pattern, indicating a transition $J=1 / 2$ to $J=1 \frac{1}{2}$. There is a little evidence that it is a combination of a new level, 34514.23 with the known level 59276.32.

A multiplet array of newly classified lines is shown in table 4. Support for the classifications is furnished by the distribution of line intensities in the group, by the repeated appearance of "constant frequency" differences, and by Zeeman patterns for the three strongest lines. It is probable that the suggested new levels are attributable to f-electron binding.

TABLE 4.-New multiplet in Xe II

\begin{tabular}{|c|c|c|c|}
\hline New level & $\begin{array}{c}\text { Suggested } \\
\text { designation }\end{array}$ & $2 \mathrm{~F}_{21 / 2} 56317.32$ & ${ }^{2} \mathrm{~F}_{31 / 2} 56154.44$ \\
\hline 33574.65 & ${ }^{2} \mathrm{~F}_{31 / 2}$ & $\begin{array}{r}(500 H l) \\
4395.77 \\
22742.77\end{array}$ & $\begin{array}{r}(2 h) \\
4427.52 \\
22579.69\end{array}$ \\
\hline 33679.22 & ${ }^{2} \mathrm{~F}_{21 / 3}$ & $\begin{array}{r}(150 H l) \\
4416.07 \\
22638.24\end{array}$ & $\begin{array}{r}(500 H) \\
4448.13 \\
22475.07\end{array}$ \\
\hline 33750.19 & ${ }^{2} \mathrm{G}_{41 / 2}$ & & $\begin{array}{r}(1000 H) \\
4462.19 \\
22404.25\end{array}$ \\
\hline
\end{tabular}

\section{XEIII-DATA AND RESULTS}

The third spectrum of xenon, which appears along with the second in condensed spark discharges, exhibits its most intense lines, barring the extreme ultraviolet lines involving the normal state, in the region between 3000 and $4000 \mathrm{~A}$. This region is rather favorable for the observation of the Zeeman effect since most gratings and photographic emulsions perform best in this range. Among these intense lines the most conspicuous are combinations of levels of the $5 s^{2} 5 p^{3}$ $\left({ }^{4} \mathrm{~S}^{\circ}\right) 6 s$ group with those of the $5 s^{2} 5 p^{3}\left({ }^{4} \mathrm{~S}^{\circ}\right) 6 p$ configuration. Next to these in intensity are the combinations of levels from the same configurations in the $\left({ }^{2} \mathrm{D}^{\circ}\right)$ family. These two types of transitions supply almost all our Zeeman patterns, inasmuch as the $\left({ }^{2} \mathrm{P}^{\circ}\right)$ family of levels gives combinations of considerably less intensity.

The observed and calculated Zeeman patterns in Xe IIr are given in table 5 along with the wave lengths, wave numbers, and appropriate designations of the respective lines. Table 5 is constructed according 
to the same plan as table 1 for Xe II. In cases where evidence afforded by this investigation indicates a different classification for a line, the revised designations appear in the table. These revisions are taken up in detail in the following discussion.

TABLE 5.-Zeeman palterns in $\mathrm{Xe} \mathrm{III}$

\begin{tabular}{|c|c|c|c|c|c|}
\hline $\begin{array}{l}\text { Wage } \\
\text { length } \\
\text { (air) }\end{array}$ & $\begin{array}{l}\text { Wave } \\
\text { number } \\
\text { (vac) }\end{array}$ & Term combination & $\begin{array}{l}\text { Observed Zee- } \\
\text { man pattern } \\
\text { Humphreys } \\
\text { and Meggers }\end{array}$ & $\begin{array}{l}\text { Observed } \\
\text { Zeeman } \\
\text { pattern } \\
\text { deBruin }\end{array}$ & Calculated Zeeman pattern \\
\hline$A$ & $\mathrm{cm-1}$ & & & & \\
\hline 3331.65 & 30006.56 & $41_{2}^{\circ}-\left({ }^{2} \mathrm{D}\right) 6 p{ }^{2} \mathrm{D}_{3}$ & $(0.00) 1.32$ & & $(0.00,0.32,0.64) 0.58,0.90$, \\
\hline $\begin{array}{l}3444.23 \\
3454.25 \\
3458.71\end{array}$ & $\begin{array}{l}29025.78 \\
28941.58 \\
28904.26\end{array}$ & $\begin{array}{l}\left({ }^{2} \mathrm{D}\right) 6 s^{3}{ }^{3} \mathrm{D}_{1}^{\mathrm{i}}-\left({ }^{2} \mathrm{D}\right) 6 p^{3}{ }^{3} \mathrm{D}_{2} \\
\left({ }^{2} \mathrm{D}\right) 6 s^{1} \mathrm{D}_{2}^{\circ}-(2 \mathrm{D}) 6 p^{1} \mathrm{D}_{2}\end{array}$ & $\begin{array}{ll}(0.00) & 1.57 \\
(0.00) & 1.01 \\
(0.83) & 1.47\end{array}$ & $(0.16) 1.03$ & $\begin{array}{l}(0.00,0.79) 0.38,1.17,1.96 \\
(0.12,0.24) 0.84,0.96,1.08, \\
1.20\end{array}$ \\
\hline 3467.20 & 28833. 49 & ( $\left.{ }^{2} \mathrm{D}\right) 5 d{ }^{3} \mathrm{D}_{3}^{\circ}-\left({ }^{2} \mathrm{D}\right) 6 p{ }^{1} \mathrm{D}_{2}$ & $(0.00) 1.37$ & $(0.00) 1.29$ & $(0.00,0.14,0.28) 0.94,1.08$, \\
\hline $\begin{array}{l}3468.19 \\
3509.78\end{array}$ & $\begin{array}{l}28825.26 \\
28483.69\end{array}$ & $\left({ }^{4} \mathrm{~S}\right) 6 s{ }^{8} \mathrm{~S}_{2}^{0}-\left({ }^{4} \mathrm{~S}\right) 6 p{ }^{3} \mathrm{P}_{1}$ & $\begin{array}{ll}(0.00) & 2.13 \\
(0.00) & 1.30\end{array}$ & & $(0.00,0.36) 1.59,1.95,2.31$ \\
\hline 3542.33 & 28221.97 & $\left({ }^{2} \mathrm{D}\right) 6 s^{3} \mathrm{D}_{3}^{\circ}-\left({ }^{2} \mathrm{D}\right) 6 p{ }^{3} \mathrm{P}_{2}$ & $(0.00) 1.28$ & $(0.00) 1.31$ & $\begin{array}{l}(0.00,0.05,0.10) 1.23,1.28, \\
1.33,1.38,1.43\end{array}$ \\
\hline $\begin{array}{l}3552.13 \\
3561.38\end{array}$ & $\begin{array}{l}\text { 28144. } 11 \\
28071.01\end{array}$ & $\begin{array}{l}411^{\circ}-4_{1} \\
41_{2}^{\circ}-\left({ }^{2} \mathrm{D}\right) 6 p 1 \mathrm{~F}_{3}\end{array}$ & $\begin{array}{ll}(0.00) & 1.12 \\
(0.00) & 1.28\end{array}$ & $(0.00) 1.07$ & $\begin{array}{l}(0.00,0.48) 0.42,0.90,1.38 \\
(0.00,0.19,0.38) 0.71,0.90, \\
1.09,1.28,1.47\end{array}$ \\
\hline $\begin{array}{l}3565.18 \\
3579.69\end{array}$ & $\begin{array}{l}28041.10 \\
27927.44\end{array}$ & $\left({ }^{2} \mathrm{D}\right) 6 s^{3} \mathrm{D}_{2}^{\circ}-\left({ }^{2} \mathrm{D}\right) 6 p{ }^{3} \mathrm{~F}_{3}$ & $\begin{array}{ll}(0.00) & 1.25 \\
(0.00) & 1.09\end{array}$ & $(0.00) 1.04$ & $\begin{array}{l}(0.00,0.10,0.20) 0.88,0.98 \\
1.08,1.18,1.28\end{array}$ \\
\hline 3583.64 & 27896.65 & $\left({ }^{2} \mathrm{D}\right) 6 s^{3} \mathrm{D}_{3}^{\circ}-\left({ }^{2} \mathrm{D}\right) 6 p^{3} \mathrm{~F}_{4}$ & $(0.00) \quad 1.19$ & $(0.00) 1.10$ & $\begin{array}{l}(0.00,0.05,0.10,0.15) 1.13 \text {, } \\
\quad 1.18,1.23,1.28,1.33,1.38, \\
\quad 1.43\end{array}$ \\
\hline $\begin{array}{l}3596.60 \\
3607.01\end{array}$ & $\begin{array}{l}27796.14 \\
27715.91\end{array}$ & $\left({ }^{2} \mathrm{D}\right) 6 s^{3} \mathrm{D}_{3}^{\circ}-\left({ }^{3} \mathrm{D}\right) 6 p{ }^{3} \mathrm{D}_{3}$ & $\begin{array}{ll}(0.00) & 0.72 \\
(0.00) & 1.27\end{array}$ & $(0.00) 0.69$ & $(0.11,0.22,0.33) 1.00,1.11$, \\
\hline 3609.44 & 27697. 26 & $\left({ }^{2} \mathrm{D}\right) 6 s^{3} \mathrm{D}_{3}^{\circ}-64$ & $(0.00) 1.28$ & & $\begin{array}{l}(0.00,0.02,0.04,0.06) 1.25 \text {, } \\
\quad 1.27,1.29,1.31,1.33,1.35 \text {, } \\
1.37\end{array}$ \\
\hline 3623.13 & 27592.61 & $\left({ }^{2} \mathrm{D}\right) 6 s^{3} \mathrm{D}_{2}^{\circ}-\left({ }^{2} \mathrm{D}\right) 6 p^{3} \mathrm{D}_{2}$ & $(0.00) 1.18$ & $(0.00) 1.20$ & $\begin{array}{l}(0.01,0.02) 1.16,1.17,1.18, \\
1.19\end{array}$ \\
\hline 3624.05 & 27585.60 & $\left({ }^{4} \mathrm{~S}\right) 6{ }^{5} \mathrm{~S}_{2}^{\circ}-\left({ }^{4} \mathrm{~S}\right) 6 p{ }^{5} \mathrm{P}_{3}$ & $(0.00) 1.18$ & $(0.00) 1.07$ & $\begin{array}{l}(0.00,0.38,0.76) \\
1.57,1.95,2.33\end{array}$ \\
\hline $\begin{array}{l}3632.14 \\
3641.00 \\
3649.56\end{array}$ & $\begin{array}{l}27524.16 \\
27457.19 \\
27392.79\end{array}$ & $\begin{array}{l}\text { (2) } 6 s^{3} \mathrm{P}_{0}^{\circ}-26_{1} \\
\left({ }^{2} \mathrm{D}\right) 6 s^{3} \mathrm{D}_{\mathrm{i}}^{0}-\left({ }^{2} \mathrm{D}\right) 6 p^{3} \mathrm{~F}_{2}\end{array}$ & $\begin{array}{ll}(0.00) & 1.51 \\
(0.00) & 1.06 \\
(0.00) & 1.35\end{array}$ & $(0.00) 1.38$ & 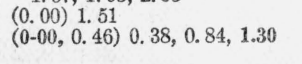 \\
\hline $\begin{array}{l}3654.63 \\
3676.63\end{array}$ & $\begin{array}{l}27354.79 \\
27191.10\end{array}$ & $\begin{array}{l}\left.{ }^{2} \mathrm{D}\right) \\
\left.\left({ }^{4} \mathrm{~S}\right) 6{ }^{3} \mathrm{~S}_{1} \mathrm{~S}_{1}^{0}-\left({ }^{2} \mathrm{D}\right) 6 p{ }^{3} \mathrm{P}^{3} \mathrm{P}\right) 6 p{ }^{3} \mathrm{P}_{0}\end{array}$ & $\begin{array}{ll}(0.00) & 1.43 \\
(0.00) & 1.76\end{array}$ & 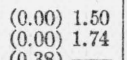 & $\begin{array}{l}(0.26) 1.30,1.56 \\
(0.00) 1.77\end{array}$ \\
\hline 3745.72 & 26689.58 & (2D) $5 d^{1} \mathrm{D}_{2}^{\circ}-\left(^{2}\right.$ & $(0.53) 0.96$ & $(0.38)-$ & $\begin{array}{l}(0.27,0.54) 0.54,0.81,1.08 \text {, } \\
1.35\end{array}$ \\
\hline $\begin{array}{l}3762.26 \\
3765.85 \\
3772.53\end{array}$ & $\begin{array}{l}26572.24 \\
26546.91 \\
26499.91\end{array}$ & $\begin{aligned}\left.{ }^{2} \mathrm{P}\right) 6 s^{3}{ }^{3} \mathrm{P} i-261 \\
25^{\circ}-36_{1}\end{aligned}$ & $\begin{array}{ll}(0.00) & 1.41 \\
(0.00) & 1.49 \\
(0.00) & 1.15\end{array}$ & 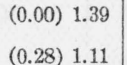 & $(0.04) 1.47,1.51$ \\
\hline 3776.30 & 27473.45 & ( $\left.{ }^{2} \mathrm{P}\right) 6 s{ }^{3} \mathrm{P}_{1}^{0}-\left({ }^{2} \mathrm{P}\right) 6 p{ }^{3} \mathrm{D}_{2}$ & $(0.00) 1.03$ & $(0.00) 0.97$ & $(0.00,0.29) 0.89,1.18,1.47$ \\
\hline $\begin{array}{l}3780.98 \\
3841.52\end{array}$ & $\begin{array}{l}26440.69 \\
26024.01\end{array}$ & $\begin{array}{l}\text { (4) } 6 s^{3} \mathrm{~S}_{1}^{1}-\left({ }^{4} \mathrm{~S}\right) 6 p{ }^{3} \mathrm{P}_{2} \\
\left.\text { ( }{ }^{2} \mathrm{D}\right) 6 s^{3} \mathrm{D}_{2}^{2}-\left({ }^{2} \mathrm{D}\right) 6 p^{3} \mathrm{~F}_{2}\end{array}$ & $\begin{array}{ll}(0.00) & 1.36 \\
(0.62) & 1.00\end{array}$ & $(0.00) 1.29$ & 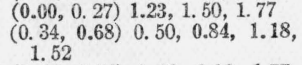 \\
\hline 3861.05 & 25892.37 & $41_{2}-\left({ }^{2} \mathrm{D}\right) 6 p^{3} \mathrm{D}_{\mathrm{z}}$ & $(0.00) \quad 1.04$ & & $(0.27,0.54) 0.63,0.90,1.77$, \\
\hline 3877.80 & 25780.54 & $\left({ }^{2} \mathrm{D}\right) 6 s^{3} \mathrm{D} \xi-\left({ }^{2} \mathrm{D}\right) 6 p^{1} \mathrm{~F}_{3}$ & $(0.47) 1.20$ & & $\begin{array}{l}(0.24,0.48,0.72) \\
\quad 1.69,1.33,1.57,1.81\end{array}$ \\
\hline $\begin{array}{l}3880.46 \\
3895.03\end{array}$ & $\begin{array}{l}25762.86 \\
25666.50\end{array}$ & (2D) $6 s^{3} \mathrm{D}_{1}^{\circ}-\left({ }^{2} \mathrm{D}\right) 6 p{ }^{3} \mathrm{D}_{1}$ & $\begin{array}{ll}(0.00) & 0.92 \\
(0.00) & 1.12\end{array}$ & & \\
\hline 3922 . & 25486 & $\left({ }^{4} \mathrm{~S}\right) 6 s^{5} \mathrm{~S}_{2}^{\circ}-\left({ }^{4} \mathrm{~S}\right) 6 p{ }^{5} \mathrm{P}_{2}$ & $(0.44) \quad 1.84$ & $(0.48) 1.74$ & $\begin{array}{l}(0.25,0.50) 1.45,1.70,1.95 \text {, } \\
2.20\end{array}$ \\
\hline $\begin{array}{l}3950.56 \\
3985.96\end{array}$ & $\begin{array}{l}25305.73 \\
25080.99\end{array}$ & $\begin{array}{c}\left({ }^{4} \mathrm{~S}\right) 6 s{ }^{5} \mathrm{~S}_{2}^{0}-\left({ }^{4} \mathrm{~S}\right) 6 p \\
23_{2}^{5}-3 P_{1}\end{array}$ & $\begin{array}{ll}(0.00) & 1.79 \\
& 1.44 \mathrm{H}\end{array}$ & & $(0.00,0.33) 1.62,1.95,2.28$ \\
\hline $\begin{array}{l}3992.85 \\
4028.58\end{array}$ & $\begin{array}{l}25037.71 \\
24815.65\end{array}$ & $\begin{array}{l}\left({ }^{2} \mathrm{D}\right) 6 s^{1} \mathrm{D}_{2}^{0}-\left({ }^{2} \mathrm{D}\right) 6 p^{3} \mathrm{P}_{1} \\
\left({ }^{2} \mathrm{D}\right) 5 d^{3} \mathrm{D}_{2}^{0}-\left({ }^{2} \mathrm{D}\right) 6 p^{3} \mathrm{P}_{2}\end{array}$ & $\begin{array}{l}(\longrightarrow \\
(\longrightarrow) \\
1.39\end{array}$ & & 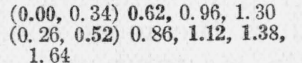 \\
\hline $\begin{array}{l}4043.21 \\
4050.05 \\
4060.43 \\
4109.07\end{array}$ & $\begin{array}{l}24725.86 \\
24684.10 \\
24621.00 \\
24329.56\end{array}$ & 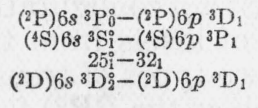 & $\begin{array}{ll}(-) & 0.65 \\
(0.00) & 1.68 \\
(0.00) & 1.01 \\
(0.00) & 1.37\end{array}$ & $\begin{array}{ll}(0.18) & 1.68 \\
(0.00) & 0.94\end{array}$ & $\begin{array}{l}(0.00) \\
(0.18) \\
(0.59\end{array}$ \\
\hline $\begin{array}{l}4142.01 \\
4145.73 \\
4176.53\end{array}$ & $\begin{array}{l}24136.08 \\
24114.43 \\
23936.60\end{array}$ & $\begin{array}{l}\text { (2P) } 6 s^{3} \mathrm{P}_{2}^{2}-26_{1} \\
\text { ('D) } 5 d^{3} \mathrm{D}_{1}^{2}-\left({ }^{2} \mathrm{D}\right) 6 p^{3} \mathrm{D}_{2} \\
\text { (2D) } 6 s^{3} \mathrm{D}_{3}^{\circ}-(2 \mathrm{D}) 6 p^{3} \mathrm{~F}_{3}\end{array}$ & $\begin{array}{ll}(\longrightarrow & 1.25 \\
(0.00) & 1.50 \\
\longrightarrow & 1.18\end{array}$ & & $\begin{array}{l}(0.00,0.67) 0.50,1.17,1.84 \\
(0.25,0.50,0.75) 0.58,0.83 \\
1.08,1.33,1.58,1.83\end{array}$ \\
\hline $\begin{array}{l}4203.92 \\
4226.96 \\
4240.24\end{array}$ & $\begin{array}{l}23780.65 \\
23651.03 \\
23576.95\end{array}$ & $\begin{array}{l}\left({ }^{2} \mathrm{D}\right) 5 d^{3} \mathrm{~S}_{1}^{\circ}-4_{1} \\
\left.17_{3}^{\circ}-\left({ }^{2} \mathrm{D}\right) 6 p^{1} \mathrm{D}_{2}\right]\end{array}$ & $\begin{array}{l}(\longrightarrow) \\
(\longrightarrow) \\
(0.00) \\
1.52 \\
1.25 ?\end{array}$ & & \\
\hline
\end{tabular}


TABLE 5.-Zeeman patterns in Xe III-Continued

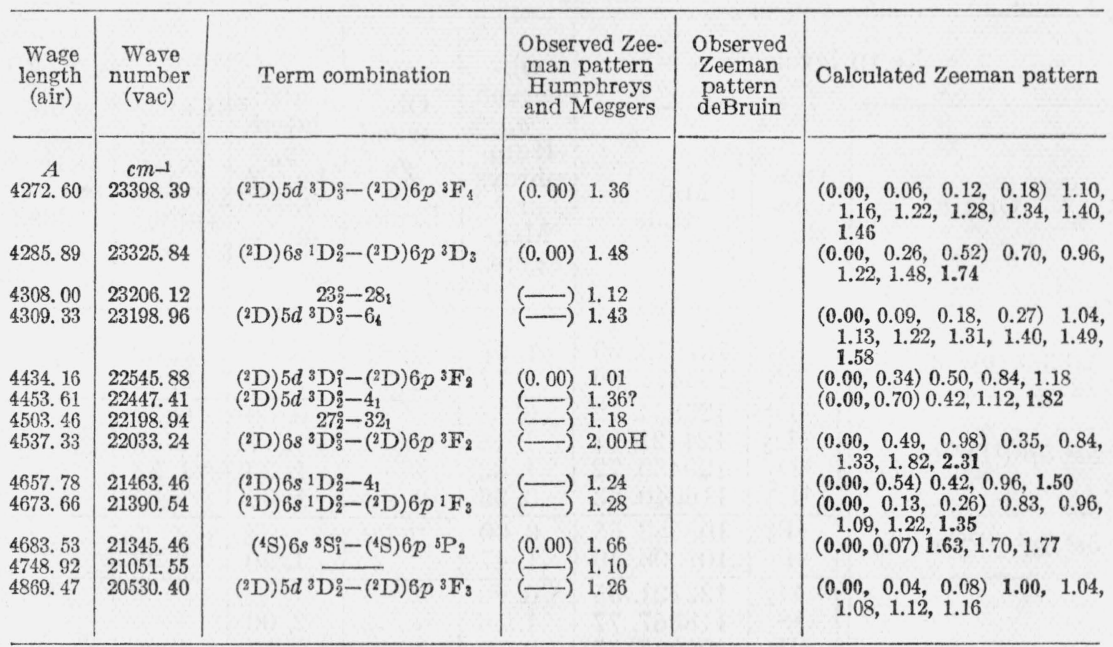

deBruin's Zeeman patterns for the lines arising from combinations of levels of the $\left({ }^{4} \mathrm{~S}^{\circ}\right)$ family are quoted from his publication on the spectral structure of doubly ionized noble gases [3].

The earlier analysis of Xe III [4] accounted for the majority of the lines clearly attributable to this spectrum and the number of energy levels found was nearly equal to the number which could be accounted for on the basis of configurations which might reasonably be expected. In many cases, however, the assignment of quantum numbers was limited to the $J$-value, and in others the complete designation was given with reservations. The reasons for this somewhat unsatisfactory state of the analysis are nonconformity with the $L S$ coupling scheme, which prevents appearance of multiplets of regular intensities and intervals; very wide level separations, as great or greater than the term separations; and mutual perturbations of levels from the same or different configurations which result in the appearance of several pairs of levels which are essentially alike as to combining properties. The usual notation for spectral terms probably means little in this kind of spectrum, but from the standpoint of simplicity, there seems to be some advantage in using it even though the coupling is not $L S$. It was expected that the Zeeman observations would permit confirmation or revision of a number of levels which were designated in somewhat arbitrary fashion. This expectation has been realized in regard to several levels which are involved in intense combinations. The outstanding line combinations among the $\left({ }^{4} \mathrm{~S}^{\circ}\right)$ family of terms yield patterns which clearly confirm the original classification [3].

Table 6 contains a list of levels for which $g$ values have been observed, together with observed and calculated $g$ 's. The calculation of $g$ 's for $J j$ coupling is made on the assumption of $L S$ coupling for the parent level. The justification for this procedure is the same in this case as it was in connection with the calculation of $g$ 's in Xe II. The designations at the left of the table are those at present regarded as most probable. A column at the extreme right gives the earlier [4] designations in cases where they differ. Discussion of these revisions follows. 
TABLE 6.-g-values-Xe III levels

\begin{tabular}{|c|c|c|c|c|c|c|c|}
\hline \multicolumn{3}{|c|}{ Xe in levels } & \multirow{2}{*}{$\begin{array}{c}\text { Ob- } \\
\text { served } \\
g, \\
\text { Hum- } \\
\text { phreys } \\
\text { and } \\
\text { Meg- } \\
\text { gers }\end{array}$} & \multirow{2}{*}{$\begin{array}{c}\text { Ob- } \\
\text { served } \\
g, \\
\text { de } \\
\text { Bruin }\end{array}$} & \multirow{2}{*}{$\begin{array}{l}\text { Calcu- } \\
\text { lated } \\
g, \\
\text { Landé } \\
\text { for- } \\
\text { mula }\end{array}$} & \multirow{2}{*}{$\begin{array}{l}\text { Calcu- } \\
\text { lated } \\
g, J j \\
\text { cou- } \\
\text { pling }\end{array}$} & \multirow{2}{*}{$\begin{array}{c}\text { For- } \\
\text { mer } \\
\text { desig- } \\
\text { nation, } \\
\text { RP898 }\end{array}$} \\
\hline Origin & $\begin{array}{c}\text { Des- } \\
\text { igna- } \\
\text { tion }\end{array}$ & $\begin{array}{l}\text { Magni- } \\
\text { tude }\end{array}$ & & & & & \\
\hline $5 s^{2} 5 p^{3}\left({ }^{4} \mathrm{~S}^{\circ}\right) 6 s$ & $\left\{\begin{array}{l}{ }^{5} \mathrm{~S}_{2}^{\mathrm{c}} \\
{ }^{3} \mathrm{~S}_{\mathrm{i}} \\
\end{array}\right.$ & $\begin{array}{l}137613.00 \\
133471.80\end{array}$ & $\begin{array}{l}\text { 1. } 95 \\
\text { 1. } 77\end{array}$ & $\begin{array}{l}\text { 1. } 87 \\
\text { 1. } 76\end{array}$ & $\begin{array}{l}\text { 2. } 00 \\
\text { 2. } 00\end{array}$ & $\begin{array}{l}\text { 2. } 00 \\
\text { 2. } 00\end{array}$ & \\
\hline $5 s^{2} 5 p^{3}\left({ }^{2} \mathrm{D}^{\circ}\right) 6 s$ & $\begin{array}{l}{ }^{3} \mathrm{D}_{1}^{\circ} \\
{ }^{3} \mathrm{D}_{2}^{\circ} \\
{ }^{3} \mathrm{D}_{3}^{\circ} \\
{ }^{1} \mathrm{D}_{2}^{\circ} \\
\end{array}$ & $\begin{array}{l}125854.72 \\
124421.51 \\
120430.72 \\
116040.63\end{array}$ & $\begin{array}{l}0.38 \\
1.18 \\
1.33 \\
0.96 \\
\end{array}$ & 0. 98 & $\begin{array}{l}0.50 \\
1.17 \\
1.33 \\
1.00\end{array}$ & $\begin{array}{l}0.50 \\
1.10 \\
1.33 \\
1.07\end{array}$ & \\
\hline $5 s^{2} 5 p^{3}\left({ }^{2} \mathrm{P}^{\circ}\right) 6 s$ & $\begin{array}{l}{ }^{3} \mathrm{P}_{0}^{\circ} \\
{ }^{3} \mathrm{P}_{\mathrm{i}}^{\circ}\end{array}$ & $\begin{array}{l}108583.55 \\
107606.40\end{array}$ & $\begin{array}{l}0.00 \\
1.47\end{array}$ & 0.00 & $\begin{array}{r}\% \\
1.50\end{array}$ & 1. $33^{\%}$ & \\
\hline $5 s^{2} 5 p^{3}\left({ }^{2} \mathrm{D}^{\circ}\right) 5 d$ & $\begin{array}{l}41_{2}^{\circ} \\
{ }^{3} \mathrm{~S}_{1}^{\circ} \\
{ }^{3} \mathrm{D}_{1}^{\circ} \\
{ }^{3} \mathrm{D}_{2}^{\circ} \\
{ }^{3} \mathrm{D}_{3}^{\circ} \\
{ }^{1} \mathrm{D}_{2}^{\circ} \\
\end{array}$ & $\begin{array}{l}122721.35 \\
118357.77 \\
120943.39 \\
117024.51 \\
115932.46 \\
113788.61 \\
\end{array}$ & $\begin{array}{l}0.90 \\
1.56 \\
0.50 \\
1.12 \\
1.22 \\
0.81 \\
\end{array}$ & 1. 19 & $\begin{array}{l}2.00 \\
0.50 \\
1.17 \\
1.33 \\
1.00 \\
\end{array}$ & & ${ }^{1 \mathrm{P}_{1}}$ \\
\hline $5 s^{2} 5 p^{3}\left({ }^{4} \mathrm{~S}^{\circ}\right) 6 p$ & $\begin{array}{l}{ }^{5} \mathrm{P}_{1} \\
{ }^{5} \mathrm{P}_{2} \\
{ }^{5} \mathrm{P}_{3} \\
{ }^{3} \mathrm{P}_{0} \\
{ }^{3} \mathrm{P}_{1} \\
{ }^{3} \mathrm{P}_{2}\end{array}$ & $\begin{array}{l}112307.29 \\
112126.40 \\
110027.40 \\
106280.64 \\
108787.71 \\
107031.06\end{array}$ & $\begin{array}{l}\text { 2. } 28 \\
\text { 1. } 70 \\
\text { 1. } 57 \\
0.00 \\
1.59 \\
1.50 \\
\end{array}$ & $\begin{array}{l}\text { 1. } 61 \\
\text { 1. } 47 \\
0.00 \\
\text { 1. } 59 \\
\text { 1. } 45\end{array}$ & $\begin{array}{r}2.50 \\
1.83 \\
1.67 \\
\% \\
1.50 \\
1.50 \\
\end{array}$ & $\begin{array}{r}2.33 \\
1.67 \\
1.67 \\
0 \% \\
1.67 \\
1.67 \\
\end{array}$ & \\
\hline $5 s^{2} 5 p^{3}\left({ }^{2} \mathrm{D}^{\circ}\right) 6 p$ & $\begin{array}{r}3 \mathrm{~F}_{2} \\
3 \mathrm{~F}_{3} \\
{ }^{3} \mathrm{D}_{2} \\
{ }^{1} \mathrm{~F}_{3} \\
4_{1} \\
6{ }_{4} \\
{ }^{3} \mathrm{D}_{3} \\
{ }^{3} \mathrm{~F}_{4} \\
{ }^{3} \mathrm{P}_{2} \\
{ }^{3} \mathrm{P}_{1} \\
{ }^{1} \mathrm{D}_{2} \\
\end{array}$ & $\begin{array}{l}98397.50 \\
96494.07 \\
96828.90 \\
94650.20 \\
94577.18 \\
92733.48 \\
92714.78 \\
92534.05 \\
92208.79 \\
91002.85 \\
87099.00 \\
\end{array}$ & $\begin{array}{l}0.84 \\
1.08 \\
1.17 \\
1.09 \\
0.42 \\
1.31 \\
1.22 \\
1.28 \\
1.38 \\
1.30 \\
1.08 \\
\end{array}$ & $\begin{array}{l}1.24 \\
1.35 \\
1.08 \\
\end{array}$ & $\begin{array}{l}1.33 \\
1.25 \\
1.50 \\
1.50 \\
1.00 \\
\end{array}$ & $\begin{array}{l}\text { 1. } 24 \\
\text { 1. } 25 \\
\text { 1. } 29 \\
\text { 1. } 10 \\
\text { 1. } 07 \\
\end{array}$ & $\begin{array}{r}{ }^{3} \mathrm{D}_{2} \\
{ }^{3} \mathrm{D}_{3} \\
{ }^{3} \mathrm{~F}_{2} \\
{ }^{2} \\
{ }^{2}{ }_{1} \\
4_{1} \mathrm{P}_{1} \\
6{ }_{3}{ }^{1} \mathrm{~F}_{3} \\
{ }^{3}{ }_{2} \\
10_{3}{ }^{3} \mathrm{~F}_{3} \\
{ }^{1} 2_{2} \\
14_{1}{ }^{3} \mathrm{P}_{1}\end{array}$ \\
\hline $5 s^{2} 5 p^{3}\left({ }^{2} \mathrm{P}^{\circ}\right) 6 p$ & $\begin{array}{l}{ }^{3} \mathrm{D}_{1} \\
{ }^{3} \mathrm{D}_{2} \\
26_{1}\end{array}$ & $\begin{array}{l}83857.65 \\
81132.93 \\
81059.42\end{array}$ & $\begin{array}{l}0.65 \\
1.18 \\
1.51\end{array}$ & 1. 38 & $\begin{array}{l}0.50 \\
1.17\end{array}$ & $\begin{array}{l}0.67 \\
1.17\end{array}$ & \\
\hline
\end{tabular}

The only important changes in designations of levels of $\mathrm{Xe}$ III have been made among the group belonging to the $5 s^{2} 5 p^{3} \cdot 6 p$ configuration and of $\left({ }^{2} \mathrm{D}^{\circ}\right)$ parentage. These levels are now capable of a much clearer interpretation. Levels previously [4] designated $2_{2}$ and $8_{2}$ must have their $J$ values increased from 2 to 3 . The former value, $2_{2}$, is now called ${ }^{1} \mathrm{~F}_{3}$ and $8_{2}$ becomes ${ }^{3} \mathrm{D}_{3}$. Similarly, the inner quantum numbers of $6_{3}$ and $10_{3}$, as listed in the earlier paper, both become 4 . After this change has been made, it seems probable that the level formerly called $10_{3}$ can now be identified as the missing level, $\left({ }^{2} \mathrm{D}^{\circ}\right) 6 p^{3} \mathrm{~F}_{4}$. There can be only one level with this inner quantum number from the $6 p$ configuration. Accordingly, $6_{4}$ must be accoun ted for by another configuration. It may belong to a term caused by the binding of an f-electron. The following changes of designation with 
retention of the same $J$ values are suggested for other levels of the same group by the observed $g$ values: $6 p^{3} \mathrm{D}_{2}$ to $6 p^{3} \mathrm{~F}_{2}, 6 p^{3} \mathrm{D}_{3}$ to $6 p^{3} \mathrm{~F}_{3}$, $6 p^{3} \mathrm{~F}_{2}$ to $6 p{ }^{3} \mathrm{D}_{2}, 12_{2}$ to $6 p^{3} \mathrm{P}_{2}$, and $14_{1}$ to $6 p^{3} \mathrm{P}_{1}$. The alternative designation, $6 p{ }^{1} \mathrm{P}_{1}$ for $4_{1}$, is abandoned on account of the low $g$ value, 0.42 , obtained for this level.

The level at 122721.35 , formerly called $\left({ }^{2} \mathrm{D}^{\circ}\right) \quad 5 d^{1} \mathrm{P}_{\mathrm{i}}$, should have a $j$ value, 2 . This rules out the former designation, so we simply give the level a number, $41_{2}^{\circ}$. Since the same level combines with 92793.33 , designated $\left({ }^{2} \mathrm{D}^{\circ}\right) 6{ }^{3} \mathrm{P}_{0}$, the $j$ value of the latter must be increased from 0 to 1 , invalidating the former classification.

Mainly on account of relative weakness of combinations within the $\left({ }^{2} \mathrm{P}^{\circ}\right)$ family, an insufficient number of patterns are available to test the $g$-sum rule. The $g$ value of the level $\left({ }^{2} \mathrm{D}^{\circ}\right) 6 s^{3} \mathrm{D}_{3}^{\circ}$ is taken to be 1.33 , according to the $g$-sum rule, because it is the only level from this configuration having a $J$ value, 3 . The $g$ value is, therefore, that given by the Landé formula.

Calculation of the $g$ 's for $J j$ coupling in the manner explained gives values in many cases identical with the Landé $g$ 's, in several others differing from the Landé $g$ 's by a relatively small amount. In the absence of a more complete set of Zeeman effect observations for $\mathrm{Xe}$ III, it is rather difficult to draw final conclusions regarding the coupling of the quantum vectors. The $g$ values for the lowest levels observed are fairly close to those given by the Landé formula, whereas the majority of the intermediate levels yield $g$ 's more closely approximating those associated with $J j$ coupling.

S. A. Goudsmit communicated some valuable suggestions in regard to the probable vector-coupling schemes in these spectra and the methods of calculating theoretical $g$ values, which the authors gratefully acknowledge.

\section{REFERENCES}

[1] C. J. Humphreys, T. L. deBruin, and W. F. Meggers, BS J. Research 6, 287 (1931) RP275.

[2] C. J. Humphreys, J. Research NBS 22, 19 (1939) RP1164.

[3] T. L. deBruin, Zeeman Verhandelingen, p. 413-423 (1935).

[4] C. J. Humphreys, J. Research NBS 16, 639 (1936) RP898.

[5] J. C. Boyce, Phys. Rev. 49, 730 (1936).

[6] L. Bloch, E. Bloch, and G. Déjardin, Ann. phys. [10] 2, 461 (1924).

[7] G. Déjardin, Ann. phys. [10] 13, 82 (1930).

[8] E. Back and A. Landé, Zeemaneffekt und Multiplettstrukten der Sroktrallinien, chap. 7 (Julius Springer, Berlin, 1925).

[9] A. Landé, Z. Physik 5, 231 (1921).

[10] C. C. Kiess and W. F. Meggers, BS J. Research 1, 641 (1928) RP23.

[11] E. Back, Z. Physik 15, 206 (1923).

[12] J. B. Green, Phys. Rev. 52, 736 (1937).

[13] W. Pauli, Z. Physik 16, 155 (1923).

[14] A. G. Shenstone and H. A. Blair, Phil. Mag. [7] 8, 765 (1929).

[15] H. Hönl, Z. Physik 31, 340 (1925).

Washington, November 3, 1939. 